TRANSACTIONS OF THE

AMERICAN MATHEMATICAL SOCIETY

Volume 361, Number 5, May 2009, Pages 2527-2566

S 0002-9947(08)04758-2

Article electronically published on December 17, 2008

\title{
FRACTIONAL SEMI-LINEAR PARABOLIC EQUATIONS WITH UNBOUNDED DATA
}

\author{
NATHAËL ALIBAUD AND CYRIL IMBERT
}

\begin{abstract}
This paper is devoted to the study of semi-linear parabolic equations whose principal term is fractional, i.e. is integral and eventually singular. A typical example is the fractional Laplace operator. This work sheds light on the fact that, if the initial datum is not bounded, assumptions on the nonlinearity are closely related to its behaviour at infinity. The sublinear and superlinear cases are first treated by classical techniques. We next present a third original case: if the associated first order Hamilton-Jacobi equation is such that perturbations propagate at finite speed, then the semi-linear parabolic equation somehow keeps memory of this property. By using such a result, locally bounded initial data that are merely integrable at infinity can be handled. Next, regularity of the solution is proved. Eventually, strong convergence of gradients as the fractional term disappears is proved for strictly convex non-linearity.
\end{abstract}

\section{INTRODUCTION}

The present paper is concerned with the following semi-linear parabolic equation:

$$
\begin{aligned}
\partial_{t} u+H(t, x, u, \nabla u)+g[u] & =0 \quad \text { in } Q_{T}:=(0, T) \times \mathbb{R}^{N}, \\
u(0, .) & =u_{0} \quad \text { on } \mathbb{R}^{N},
\end{aligned}
$$

where $\nabla u$ denotes the gradient with respect to (wrt for short) $x, H$ is a continuous first order non-linearity (or Hamiltonian) and $g[u]$ denotes a Lévy operator. An important example of a Lévy operator is the fractional Laplacian, i.e. the pseudodifferential operator defined by the symbol $|\xi|^{\lambda}, 0<\lambda<2$ : for all Schwartz function $\varphi$,

$$
g[\varphi](x):=\mathcal{F}^{-1}\left(|\cdot|^{\lambda} \mathcal{F} \varphi\right),
$$

where $\mathcal{F}$ denotes the Fourier transform. In general, Lévy operators $g[\varphi]$ have the following integral form:

$$
g[\varphi](x)=-\int_{\mathbb{R}^{N}}\left(\varphi(x+z)-\varphi(x)-\frac{\nabla \varphi(x) \cdot z}{1+|z|^{2}}\right) d \mu(z),
$$

where $\mu$ denotes the Lévy measure, i.e. a non-negative Radon measure on $\mathbb{R}^{N}$ such that $\int \min \left(1,|z|^{2}\right) d \mu(z)<+\infty$. It is convenient to write

$$
\mu=\mu_{b}+\mu_{s}
$$

Received by the editors May 3, 2007.

2000 Mathematics Subject Classification. Primary 35B65, 35D05, 35B05, 35K65, 35S30.

Key words and phrases. Semi-linear equation, Lévy operator, fractional Laplacian, unbounded data, unbounded solutions, viscosity solution, finite-infinite propagation speed, regularity, nonlocal vanishing viscosity method, convergence of gradients.

(C)2008 American Mathematical Society 
where $\mu_{b}$ is bounded and supported outside of the unit ball $B$, and $\mu_{s}$ is eventually singular $\left(\int|z|^{2} d \mu_{s}(z)<+\infty\right)$ and supported in $B$. In the case of the fractional Laplacian, the derivative of $\mu$ wrt the Lebesgue measure is $c_{N}(\lambda)|z|^{-N-\lambda}, c_{N}(\lambda)>$ 0 . Hence, the first order Hamilton-Jacobi equation is perturbed by a singular integral term. We refer to (1.1) as a fractional semi-linear parabolic equation.

In many ways, Lévy operators generalize diffusion operators. They are indeed the infinitesimal generators of Lévy (jump) processes, and Brownian motions make part of this large class of stochastic processes. In particular, they satisfy maximum principles 16. They appear in many models from mathematical finance [15, biology [29, 31], combustion theory [13], continuum mechanics [4, 20], fluid mechanics 32, 14, 24], physical science [35, etc. We can explain their extensive use in models by two fundamental facts. First, their distribution functions decay as a power law (instead of exponentially fast). Secondly, Lévy operators can appear when dimension is reduced; for instance, in [4, the geometric motion of a curve of defects in a crystal is obtained by considering the Dirichlet to Neumann operator associated with the elliptic linear elasticity equation.

The study of fractional non-linear equations has attracted a lot of attention for more than thirty years, and the literature is now rapidly growing as far as fractional non-linear elliptic and parabolic equations are concerned. A relevant example comes from the study of stochastic control of jump processes. Equation (1.1) can be interpreted as the Bellman equation of such an optimal control problem if there is no control on the jumps. Maximum principle techniques are used for instance in [9, 23] in order to construct solutions in Sobolev spaces. Soner 34 first used the weak notion of viscosity solution to deal with more general non-linear equations. Indeed, viscosity solution theory provides a good framework, and there is an important literature about it; see for instance [33, 3, 28, 8, [5] and the references therein. A second class of non-linear equations is the one composed with fractional scalar conservation laws [10, 11, 19, 1, 2. We would also like to mention the books by Garroni and Menaldi 21, 22, about linear equations.

The aim of this paper is to study equation (1.1) in full detail. In particular, existence, uniqueness and regularity of solutions are investigated. Because of several applications we have in mind, one of our goal, is to be able to deal with unbounded initial data $u_{0}$ (see (1.2)). For instance, if equation (1.1) is seen as the Bellman equation associated with a control problem involving jump processes, a natural initial condition is $u_{0}(x)=\max (0, x-K)$.

In 25, such a Cauchy problem is studied with $u_{0} \in L^{\infty}$ in the case of the fractional Laplacian with $\lambda>1$. Regularizing effects are exhibited: with bounded Lipschitz continuous initial data, the solution turns out to be $C^{2}$ in space and $C^{1}$ in time. We will see that these results are easily extended to the case of sublinear initial data, i.e. functions $u_{0}$ for which there exists $C>0$ such that for all $x \in \mathbb{R}^{N}$

$$
\left|u_{0}(x)\right| \leq C(1+|x|) \text {. }
$$

Computations next suggest that even superlinear initial conditions can be handled. Precisely, if $u_{0}$ satisfies for all $x \in \mathbb{R}^{N}$

$$
\left|u_{0}(x)\right| \leq C\left(1+|x|^{\lambda^{\prime}}\right)
$$

for a suitable $\lambda^{\prime}>1$ and constant $C>0$ (we say that such a function is superlinear), assumptions on the non-linearity can be modified in order to prove a comparison principle; roughly speaking, the dependence in $x$ of $H$ is relaxed and the one in $p$ 
is strengthened. See the introduction of Section 3 for a more detailed discussion about assumptions on $H$. Jakobsen and Karlsen 28] developed a general theory for second order parabolic non-linear integro-differential equations. In particular, they establish comparison results and continuous dependence estimates. Because of the dependence of $H$ on the Hessian of $u$, their arguments are more involved. In our case, classical techniques work with minor modifications and under more general assumptions (a modulus of continuity appear in assumptions (B2) and (B3) instead of a Lipschitz constant; (B2) can be local in $x$ and $u$ ).

As far as the superlinear case is concerned, condition (1.5) ensures in particular that the following uniform integrability condition is satisfied by the continuous function $u_{0}$ :

$$
\int\left(\sup _{B_{1}(z)} u_{0}\right) d \mu_{b}(z)<+\infty
$$

where $\mu_{b}$ is the bounded part of $\mu$ (it is introduced in (1.4)). The third class of unbounded initial data $u_{0}$ that we can treat corresponds to assuming that $u_{0}$ satisfy the uniform integrability condition (1.6). If assumptions of the superlinear case are strenghtened, existence and (strong) uniqueness can be proved for (1.1). Moreover, a local $L^{\infty}$ bound is derived. We next make assumptions on the non-linearity ensuring that the associated first order Hamilton-Jacobi equation (with no integral operator) propagates perturbations of $u_{0}$ with finite speed. This means that the solution at a point $(t, x)$ only depends on the value of the initial condition on a ball centered at $x$ and of radius proportional to $t$ 30, 26. Following an original idea of the first author [1, we show that, for an initial condition satisfying (1.6), the solutions of (1.1) keep memory of this finite speed property, even if perturbations now propagate with infinite speed. Precisely, we prove that if $u$ and $v$ are solutions of (1.1) associated with initial data $u_{0}$ and $v_{0}$, then

$$
u(t, x)-v(t, x) \leq K(t, \cdot) * \sup _{|y-\cdot| \leq C_{2} t}\left(u_{0}(y)-v_{0}(y)\right)(x),
$$

where $K$ is the kernel of the operator $g$. Following [1], such a property is referred to as a finite-infinite propagation speed property. Let us make an important remark: this last result only relies on the fact that the perturbation operator has a positive kernel (or Green function) and that it can also be applied to any Lévy operator, including the Laplacian. In this latter case, we recover (more or less) classical results where $\left(1+|z|^{N+\lambda}\right)^{-1}$ is replaced with $\exp \left(-|z|^{2}\right)$.

We previously mentioned that the fractional Laplacian has a regularizing effect for $\lambda>1$. The results we obtain here in the sublinear framework are in the spirit of those of [25], and the proofs rely on them. They are obtained in the case of a Lipschitz continuous initial datum $u_{0}$. As far as superlinear initial conditions are concerned, we give two different results: the first one asserts the $C^{1}$ regularity in space of the solutions for superlinear initial conditions that are locally Lipschitz continuous and for a non-linearity whose Lipschitz constant in $p$ can depend linearly on $|x|$. If this assumption is strengthened, then $C^{1,1}$ in space solutions are obtained even for initial conditions that are not locally Lipschitz continuous. 
We finally consider the case where the non-local part of equation (1.1) vanishes. Precisely, we consider

$$
\begin{aligned}
\partial_{t} u^{\varepsilon}+H\left(t, x, u^{\varepsilon}, \nabla u^{\varepsilon}\right)+\varepsilon g\left[u^{\varepsilon}\right] & =0 \quad \text { in } Q_{T}, \\
u^{\varepsilon}(0, .) & =u_{0} \quad \text { on } \mathbb{R}^{N}
\end{aligned}
$$

with $u_{0}$ (locally) Lipschitz continuous, and we let $\varepsilon$ go to 0 . The viscosity solution theory ensures that the regular solution $u^{\varepsilon}$ converges as $\varepsilon \rightarrow 0$ locally uniformly towards the (locally) Lipschitz continuous solution $u^{0}$ of (1.7) with $\varepsilon=0$. Hence, the sequence $\left\{\nabla u^{\varepsilon}\right\}_{\varepsilon>0}$ converges towards $\nabla u^{0}$ in the distribution sense, but it is not clear if strong convergence in $L_{\text {loc }}^{p}, p \geq 1$, can be obtained. We prove it in the case where the non-linearity is strictly convex with respect to the gradient variable by using Young measure techniques. Let us mention that the result about the convergence of gradients remains true if the non-local operator is replaced with the Laplacian. Strong convergence of sequences of gradients appear in [18] in the context of non-linear elliptic equations in the divergence form. To the best of our knowledge, there are very few results in the literature as far as Hamilton-Jacobi equations are concerned, and we did not find any references. To finish, we shed some light on the fact that the proof only relies on local gradient bounds and the strict convexity of $H$ in $p$.

Let us mention that we decided to present most of our results with the classical fractional Laplacian instead of a general Lévy operator to avoid blinding technicality. If general Lévy operators are at stake, most of the results can be adapted if assumptions are properly translated. For instance, condition $\lambda^{\prime} \in(0, \lambda)$ in the suband superlinear cases should be replaced with

$$
\int|z|^{\lambda^{\prime}} d \mu_{b}(z)<+\infty
$$

and the results of Subections 3.1 and 3.2 remain true under this assumption. As far as regularity results of Section 4 are concerned, the techniques of [25] we use there only rely on the fact that the kernel of the fractional Laplacian satisfies

$$
\sup _{t \in[0, T]} t^{\frac{1}{\lambda}} \int_{\mathbb{R}^{N}}\left(1+|x|^{\lambda^{\prime}}\right)|\nabla K(t, x)| d x<+\infty
$$

for all $\lambda^{\prime} \in[0, \lambda)$. As far as the integrable case is concerned, strong uniqueness result and existence for instance rely on the construction of barrier functions (see Lemma 2.5.2). We are convinced that such a construction could be done for a large class of Lévy operators, if not for all of them. See also [5] for further discussion.

The paper is organized as follows. In Section 2, norms and function spaces that are used throughout the paper are introduced. Next, the definition of a viscosity solution for (1.1) is given and technical results related to this notion of weak solutions are stated. We also recall in this section some useful properties of the kernel associated with the non-local operator. Section 3 is devoted to existence and uniqueness results and gradient estimates in the three frameworks described above. In Section 4, we investigate regularity issues in the sublinear and the superlinear cases. Next, a non-local vanishing viscosity method is considered in the last section; in particular, gradients are proved to converge strongly in $L_{\mathrm{loc}}^{p}, p \geq 1$, in the case where the non-linearity is strictly convex with respect to the gradient variable. Eventually, we gathered in the appendices several (more or less) classical proofs for 
the reader's convenience; a list of the different assumptions used throughout this paper is also given just before the references.

Notation. We let $|\cdot|$ denote the Euclidian norm, $B_{R}(x)$ denote the closed ball of $\mathbb{R}^{N}$ centered at $x$ of radius $R, B_{R}$ denote the ball $B_{R}(0)$ and $B$ denote the unit ball $B_{1}$. For any real $r$, we let $r^{+}:=\max \{r, 0\}$ denote the positive part of $r$ and $r^{-}:=(-r)^{+}$its negative part. A modulus (of continuity) is a non-decreasing function $m: \mathbb{R}^{+} \rightarrow \mathbb{R}^{+}$such that $\lim _{r \rightarrow 0} m(r)=0$ and $m(r+s) \leq m(r)+m(s)$ for every $r, s \geq 0$.

For a Lévy measure $\mu$ with a density $M$ with respect to the Lebesgue measure, $\mu_{b}$ denotes the non-negative bounded measure whose density is $\mathbf{1}_{B^{c}} M$. The space of integrable function on $\mathbb{R}^{N}$ wrt $\mu_{b}$ is denoted by $L^{1}\left(\mathbb{R}^{N}, \mu_{b}\right)$. If now $\mu_{s}$ denotes $\mu-\mu_{b}$, one can check that (1.4) holds true.

\section{Preliminaries}

This section is devoted to definitions and technical tools that are used throughout the paper. We first introduce notation for norms and function spaces (Subsection 2.1), and we next recall the definition of viscosity solutions for fractional semi-linear parabolic equations (Subsection 2.2). In Subsection 2.3 are gathered technical results related to stability issues. Subsection 2.4 is devoted to the properties of the kernel $K$ (or Green function) associated with the Lévy operator $g[\cdot]$ used in the proofs of the main results. In the last subsection, we define the notion of uniform integrability related to condition (1.6) and gives some basic properties.

Let us mention that we assume throughout the paper that the function $H$ : $[0, T] \times \mathbb{R}^{N} \times \mathbb{R} \times \mathbb{R}^{N} \rightarrow \mathbb{R}$ is continuous and that $\lambda \in(0,2)$.

\subsection{Norms and function spaces.}

Data. Consider functions $u_{0}: \mathbb{R}^{N} \rightarrow \mathbb{R}$. The space of continuous (resp. uniformly continuous) functions $u_{0}$ is denoted by $C\left(\mathbb{R}^{N}\right)$ (resp. $U C\left(\mathbb{R}^{N}\right)$ ). For $u_{0} \in C\left(\mathbb{R}^{N}\right)$, $\lambda^{\prime} \in(0, \lambda)$ and $\beta \in(1, \lambda)$ (if $\lambda>1$ ), define the norms and semi-norm

$$
\begin{aligned}
\left\|u_{0}\right\|_{0, \lambda^{\prime}} & :=\sup _{x \in \mathbb{R}^{N}} \frac{\left|u_{0}(x)\right|}{1+|x|^{\lambda^{\prime}}}, \\
{\left[u_{0}\right]_{1, \beta} } & :=\sup _{x, y \in \mathbb{R}^{N}, x \neq y} \frac{\left|u_{0}(x)-u_{0}(y)\right|}{\left(1+|x|^{\beta-1}+|y|^{\beta-1}\right)|x-y|}, \\
\left\|u_{0}\right\|_{1, \beta} & :=\left\|u_{0}\right\|_{0, \beta}+\left[u_{0}\right]_{1, \beta}
\end{aligned}
$$

and the associated function spaces

$$
\begin{aligned}
& C_{\lambda^{\prime}}^{0}\left(\mathbb{R}^{N}\right):=\left\{u_{0} \in C\left(\mathbb{R}^{N}\right):\left\|u_{0}\right\|_{0, \lambda^{\prime}}<+\infty\right\}, \\
& C_{\beta}^{0,1}\left(\mathbb{R}^{N}\right):=\left\{u_{0} \in C\left(\mathbb{R}^{N}\right):\left\|u_{0}\right\|_{1, \beta}<+\infty\right\} .
\end{aligned}
$$

In discussions, $C_{\lambda^{\prime}}^{0}\left(\mathbb{R}^{N}\right)$ and $C_{\beta}^{0,1}\left(\mathbb{R}^{N}\right)$ are respectively denoted $C_{\lambda^{\prime}}^{0}$ and $C_{\beta}^{0,1}$ for short.

Solutions. Consider functions $u: \bar{Q}_{T} \rightarrow \mathbb{R}$, where $\bar{Q}_{T}:=[0, T] \times \mathbb{R}^{N}$. Define the norms

$$
\|u\|_{0, \lambda^{\prime}}:=\sup _{t \in[0, T]}\|u(t, \cdot)\|_{0, \lambda^{\prime}} \quad \text { and } \quad\|u\|_{1, \beta}:=\sup _{t \in[0, T]}\|u(t, \cdot)\|_{1, \beta}
$$


and the associated function spaces

$$
\begin{aligned}
& C_{\lambda^{\prime}}^{0}\left(\bar{Q}_{T}\right):=\left\{u \in C\left(\bar{Q}_{T}\right):\|u\|_{0, \lambda^{\prime}}<+\infty\right\}, \\
& C_{\beta}^{0,1}\left(\bar{Q}_{T}\right):=\left\{u \in C\left(\bar{Q}_{T}\right):\|u\|_{1, \beta}<+\infty\right\} .
\end{aligned}
$$

In discussions, $C_{\lambda^{\prime}}^{0}\left(\bar{Q}_{T}\right)$ and $C_{\beta}^{0,1}\left(\bar{Q}_{T}\right)$ are respectively denoted $C_{\lambda^{\prime}}^{0}$ and $C_{\beta}^{0,1}$ for short.

2.2. Viscosity solutions for (1.1). Let us introduce the notion of viscosity solution to (1.1). Even if this weak notion of solution is nowaways well known, we would like to recall some basic facts about it. First, a weak notion of differentiability is obtained by "touching" (from below or above) a semi-continuous function $u$ with smooth test-functions $\phi$. Second, it is very easy to pass to the limit in non-linear terms with this notion of solution [6]; in other words, good stability results are available. Third, it is possible (and we will do so later) to prove strong uniqueness results; they are obtained under the form of comparison principles. To finish, existence of solutions is generally proved by using Perron's method [27]. For a general introduction, the interested reader is referred to the survey [17] or to the book [7] for instance.

We now turn to the definition of viscosity solution for equation (1.1). Following 25, we will use an appropriate notion of parabolic sub- and supergradients to define viscosity solutions of first order integro-differential equations.

Definition 2.2.1 (Parabolic supergradient). Let $u: \bar{Q}_{T} \rightarrow \mathbb{R}$ be upper semicontinuous (usc for short). Then, $(a, p) \in \mathbb{R} \times \mathbb{R}^{N}$ is a (parabolic) supergradient of $u$ at $(t, x) \in \bar{Q}_{T}$ if there exist $r>0$ and $\sigma>0$ such that for all $s \in[0, T]$ and all $y \in B_{r}(x)$ :

$$
u(s, y) \leq u(t, x)+a(s-t)+p \cdot(y-x)+\sigma\left(|y-x|^{2}\right)+o(|s-t|),
$$

where $\frac{o(l)}{l} \rightarrow 0$ as $l \rightarrow 0^{+}$.

In the following, $\partial^{P} u(t, x)$ denotes the set of all supergradients of $u$ at $(t, x)$ and it is referred to as the superdifferential of $u$ at $(t, x)$. If $u$ is lower semicontinuous (lsc for short), we then define (parabolic) subgradients and subdifferentials by $\partial_{P} u(t, x):=-\partial^{P}(-u)(t, x)$.

We can now introduce the notion of viscosity solution to (1.1) in the spirit of [5]. We do it for the following general class of functions:

$$
\begin{aligned}
\mathcal{C}^{+(-)}:=\left\{u: \bar{Q}_{T} \rightarrow \mathbb{R} \text { s.t. } \exists \Phi \in C^{2}\left(\mathbb{R}^{N}\right) \cap L^{1}\left(\mathbb{R}^{N}, \mu_{b}\right),\right. \\
\\
\left.\forall(t, x) \in \bar{Q}_{T}, u^{+(-)}(t, x) \leq \Phi(x)\right\},
\end{aligned}
$$

where $\mu_{b}$ appears in (1.4) (see also the end of the Introduction). We will consider solutions that lie in $\mathcal{C}:=\mathcal{C}^{+} \cap \mathcal{C}^{-}$.

Let us mention that we will consider different subspaces of $\mathcal{C}$ : sublinear functions, superlinear ones and uniformly integrable ones (see below for a definition).

We now can give the definition of a viscosity solution of (1.1).

Definition 2.2.2 (Viscosity solutions for (1.1)). (1) A usc function $u \in \mathcal{C}^{+}$is a viscosity subsolution of (1.1) if for all $(t, x) \in Q_{T}$ and all $(a, p) \in \partial^{P} u(t, x)$,

$$
a+H(t, x, u(t, x), p)+g[u](t, x, p) \leq 0,
$$

where $g[u](t, x, p):=-\int_{\mathbb{R}^{N} \backslash\{0\}}\left(u(t, x+z)-u(t, x)-\frac{p \cdot z}{1+|z|^{2}}\right) d \mu(z)$. 
(2) An lsc function $u \in \mathcal{C}^{-}$is a viscosity supersolution of (1.1) if for all $(t, x) \in$ $Q_{T}$ and all $(a, p) \in \partial_{P} u(t, x)$,

$$
a+H(t, x, u(t, x), p)+g[u](t, x, p) \geq 0 .
$$

(3) A continuous function $u \in \mathcal{C}$ is a viscosity solution of (1.1) if it is both a viscosity sub- and supersolution.

Remarks 2.1. (1) Notice that the integral term $g[u](t, x, p)$ makes sense since its integrand is $\mu$-quasi-integrable, thanks to (2.1) and to the integrability condition on $u$ at infinity. Actually, it is $\mu$-integrable because of (2.2). Such an idea already appears in 25 . See also $[5$, for a complete discussion.

(2) If $\lambda \in(0,1)$, then we obtain an equivalent definition by replacing the notion of (parabolic) supergradients with the one of first order Fréchet supergradients.

2.3. Stability results. This subsection is devoted to classical and technical results related to stability issues. It contains two technical lemmata that are used in the proofs of comparison principles. It also contains a discontinuous stability result that permits us to pass to the limit in the equation in a very weak sense. Sketches of the proofs of these results are given in Appendix A. The reader can easily skip this technical subsection since it is not necessary in the understanding of the statements of the main results.

When proving comparison principles, we must show that subsolutions $u$ lie below supersolutions $v$. In order to do so, we consider $M=\sup (u-v)$ and, in order to get viscosity inequalities, the number of variables is doubled and this doubling is penalized. Lemmata 2.3.1 and 2.3.2 are related to the doubling of the time variable.

In order to state and use these lemmata, we need to introduce the closure of suband superdifferentials.

Definition 2.3.1 (Limiting subgradients). For $u$ usc, $\bar{\partial}^{P} u(t, x)$ denotes the set of $(a, p) \in \mathbb{R} \times \mathbb{R}^{N}$ such that there exist $r, \sigma>0$ and a sequence $\left(\left(t_{n}, x_{n}, a_{n}, p_{n}, r_{n}, \sigma_{n}\right)\right)_{n \in \mathbb{N}}$ such that $\left(a_{n}, p_{n}\right) \in \partial^{P} u\left(t_{n}, x_{n}\right)$, with (2.1) holding true with the constants $r_{n}, \sigma_{n}>0$, and such that $\left(t_{n}, x_{n}, u\left(t_{n}, x_{n}\right), a_{n}, p_{n}, r_{n}, \sigma_{n}\right) \rightarrow$ $(t, x, u(t, x), a, p, r, \sigma)$ as $n \rightarrow+\infty$.

We then define $\bar{\partial}_{P} u(t, x):=-\bar{\partial}^{P}(-u)(t, x)$ for $u$ lsc.

Lemma 2.3.1. If $u \in \mathcal{C}^{+}$is a viscosity subsolution of (1.1), then (2.2) holds true for all $(t, x) \in(0, T] \times \mathbb{R}^{N}$ and all $(a, p) \in \bar{\partial}^{P} u(t, x)$.

Remark 2.1. Remark that, passing to the limit in the equation, we conclude that the integrand of the integral term $g[u](t, x, p)$ is $\mu$-quasi-integrable with $g[u](t, x, p)<$ $+\infty$. Nevertheless, it could occur that $g[u](t, x, p)=-\infty$; in that case, (2.2) holds true in $\mathbb{R} \cup\{-\infty\}$.

Lemma 2.3.2. Let $u \in \mathcal{C}^{+}$and $v \in \mathcal{C}^{-}$be, respectively, a sub- and a supersolution of equation (1.1). Let $\phi:[0, T] \times \mathbb{R}^{N} \times \mathbb{R}^{N} \rightarrow \mathbb{R}$ be smooth and such that the supremum

$$
M:=\sup _{[0, T] \times \mathbb{R}^{N} \times \mathbb{R}^{N}}(u(t, x)-v(t, y)-\phi(t, x, y))
$$

is achieved at some point $(\bar{t}, \bar{x}, \bar{y})$. There then exist $a, b \in \mathbb{R}$ such that

$$
\left(a, \nabla_{x} \phi(\bar{t}, \bar{x}, \bar{y})\right) \in \bar{\partial}^{P} u(\bar{t}, \bar{x}),\left(b,-\nabla_{y} \phi(\bar{t}, \bar{x}, \bar{y})\right) \in \bar{\partial}_{P} v(\bar{t}, \bar{y})
$$


and $a-b=\partial_{t} \phi(\bar{t}, \bar{x}, \bar{y})$. Moreover,

$$
\begin{aligned}
& g[v]\left(\bar{t}, \bar{y},-\nabla_{y} \phi(\bar{t}, \bar{x}, \bar{y})\right)-g[u]\left(\bar{t}, \bar{x}, \nabla_{x} \phi(\bar{t}, \bar{x}, \bar{y})\right) \\
& \quad \leq \int_{\mathbb{R}^{N}}\left(\phi(\bar{t}, \bar{x}+z, \bar{y}+z)-\phi(\bar{t}, \bar{x}, \bar{y})-\frac{\left(\nabla_{x}+\nabla_{y}\right) \phi(\bar{t}, \bar{x}, \bar{y}) \cdot z}{1+|z|^{2}}\right) d \mu(z) .
\end{aligned}
$$

Remark 2.2. Following the previous remark, (2.3) holds true in $\mathbb{R} \cup\{+\infty\}$. But this lemma will be used with $\phi$ such that the last integral term of (2.3) is finite; hence, the integral terms $g[v]\left(\bar{t}, \bar{y}, \nabla_{y} \phi(\bar{t}, \bar{x}, \bar{y})\right)$ and $g[u]\left(\bar{t}, \bar{x}, \nabla_{x} \phi(\bar{t}, \bar{x}, \bar{y})\right)$ will also be finite.

We now turn to the discontinuous stability result. When dealing with non-linear equations, it is crucial to be able to pass to the limit in the non-linear terms in an appropriate sense. It turns out that it is very easy to do so when working with viscosity solutions. Indeed, Barles and Perthame [6] remarked that the socalled relaxed lower/upper limits of a family of super-/subsolutions is still a super/subsolution (see below for a precise definition). It is also classical to prove that the supremum of a family of subsolutions is a subsolution of (1.1). This result is useful to prove existence by the classical Perron's method.

Let us make precise the definition of relaxed semi-limits. Let us recall that if $\mathcal{O}$ is a non-empty subset of $\mathbb{R}^{M}$, then the relaxed upper limit of a sequence of functions $u_{n}: \mathcal{O} \rightarrow \mathbb{R}$ is defined as follows:

$$
\lim \sup ^{*} u_{n}(x)=\limsup _{n \rightarrow+\infty, \mathcal{O} \ni y \rightarrow x} u_{n}(y) .
$$

The relaxed lower limit is defined by $\liminf _{*} u_{n}:=-\lim \sup ^{*}\left(-u_{n}\right)$. We then have the following result.

Proposition 2.1 (Discontinuous stability). Let $\left(u_{n}\right)_{n}$ be a sequence of subsolutions of (1.1) such that there exists $\Phi \in C^{2}\left(\mathbb{R}^{N}\right) \cap L^{1}\left(\mathbb{R}^{N}, \mu_{b}\right)$ with

$$
\forall n \in \mathbb{N}, \forall(t, x) \in \bar{Q}_{T}, u_{n}^{+}(t, x) \leq \Phi(x) .
$$

Then $\lim \sup ^{*} u_{n}$ is a viscosity subsolution of (1.1). Moreover,

$$
\left(\lim \sup ^{*} u_{n}\right)(0, .) \leq \lim \sup ^{*}\left(u_{n}(0, .)\right) \text {, }
$$

where the relaxed upper semi-limit of the left-hand side is computed wrt time and space while the one of the right-hand side is only computed wrt the space variable.

Remarks 2.2. (1) An analogous result for supersolution can be easily stated and proved. Hence one can pass to the limit in (1.1) wrt the local uniform convergence of sequences of solutions.

(2) Inequality (2.4) implies that if $u_{0}$ is an initial condition, then the notion of subsolution of (1.1)-(1.2) (the initial condition being replaced with $u(0,$. $u_{0}$ ) is stable by passing to the upper semi-limit. Such a property will be used to prove the convergence of a sequence of solutions under only $L_{l o c}^{\infty}$ estimates by the so-called technique of the relaxed semi-limits (see [6]).

2.4. The kernel associated with the non-local operator. The semi-group generated by $g$ is formally given by the convolution with the kernel defined for $t>0$ by $K(t, \cdot):=\mathcal{F}^{-1}\left(e^{-t|\cdot|^{\lambda}}\right)$. It is well-known that $K$ satisfies the following 
properties (see [19] and the item (1) of the remarks below):

$$
\begin{gathered}
K(1, \cdot) \in C_{b}^{\infty}\left(\mathbb{R}^{N}\right) \cap W^{\infty, 1}\left(\mathbb{R}^{N}\right) \text { is positive and } \int_{\mathbb{R}^{N}} K(1, \cdot)=1, \\
K(t, x)=t^{-N / \lambda} K\left(1, t^{-1 / \lambda} x\right), \\
K(t, \cdot) * K(s, \cdot)=K(t+s, \cdot) .
\end{gathered}
$$

To deal with unbounded solutions, we need the following estimates:

$$
\begin{array}{r}
\int_{0}^{T} \int_{\mathbb{R}^{N}}\left(1+|x|^{\lambda^{\prime}}\right) K(t, x) d x<+\infty, \\
\mathcal{K}_{\lambda^{\prime}}:=\sup _{t \in[0, T]} t^{\frac{1}{\lambda}} \int_{\mathbb{R}^{N}}\left(1+|x|^{\lambda^{\prime}}\right)|\nabla K(t, x)| d x<+\infty,
\end{array}
$$

for all $\lambda^{\prime} \in[0, \lambda)$. These estimates follow immediately from the homogeneity property and the lemma below.

Lemma 2.4.1 ([12]). Consider $\lambda \in(0,2)$. There exists $C_{\lambda}>0$ such that, as $|x| \rightarrow+\infty$, we have $K(1, x) \sim C_{\lambda}|x|^{-N-\lambda},|\nabla K(1, x)|=\mathcal{O}\left(|x|^{-N-\lambda-1}\right)$ and $|g[K(1, \cdot)](x)|=\mathcal{O}\left(|x|^{-N-2 \lambda}\right)$.

Remarks 2.3. (1) In [19, it is only proved that $K$ is non-negative. But in [2], it is proved that it is radially decreasing. Combining this result with the ones of Lemma 2.4 .1 permits us to conclude that $K$ cannot vanish.

(2) Actually, we have results similar to the ones of Lemma 2.4.1 for the derivatives of all order.

2.5. Uniformly integrable functions. In this subsection, we give the definition of uniform integrability of a function (with respect to $\mu_{b}$ ) and give some basic properties.

Definition 2.5.1 (Uniformly integrable function). Let $u_{0}: \mathbb{R}^{N} \rightarrow \mathbb{R}$ be locally bounded. We say that $u_{0}$ is $\mu_{b}$-uniformly integrable if there exists $R>0$ such that

$$
\int_{\mathbb{R}^{N}} \sup _{B_{R}(z)}\left|u_{0}\right| d \mu_{b}(z)<+\infty .
$$

Remark 2.3. We will use this notion for locally bounded functions $u: \bar{Q}_{T} \rightarrow \mathbb{R}$. In this case, we investigate whether or not $\sup _{t \in[0, T]}|u(t, \cdot)|$ is $\mu_{b}$-uniformly integrable. If yes, we simply say that $u$ is $\mu_{b}$-uniformly integrable in space uniformly wrt time.

Here is a result that makes precise the structure of such functions.

Lemma 2.5.1. If $u_{0}$ is $\mu_{b}$-uniformly integrable, then (2.7) holds true for all $R>0$.

Proof. Let $R>r>0$. For $z \in \mathbb{R}^{N}$, there exists $y_{z} \in B_{R}(z)$ such that $B_{\frac{r}{2}}\left(y_{z}\right) \subset$ $B_{R}(z)$ and

$$
\sup _{B_{R}(z)}\left|u_{0}\right|=\sup _{B_{\frac{r}{2}}\left(y_{z}\right)}\left|u_{0}\right| .
$$

For $y \in B_{\frac{r}{2}}\left(y_{z}\right)$, we have $B_{\frac{r}{2}}\left(y_{z}\right) \subset B_{r}(y)$ and $\frac{1}{1+|z|^{N+\lambda}} \leq \frac{C(R, N, \lambda)}{1+|y|^{N+\lambda}}$. Hence,

$$
\frac{\sup _{B_{R}(z)}\left|u_{0}\right|}{1+|z|^{N+\lambda}} \leq C(R, N, \lambda) \frac{\sup _{B_{r}(y)}\left|u_{0}\right|}{1+|y|^{N+\lambda}},
$$


and integrating wrt $y \in B_{\frac{r}{2}}\left(y_{z}\right)$ yields:

$$
\begin{aligned}
\left|B_{\frac{r}{2}}\right| \frac{\sup _{B_{R}(z)}\left|u_{0}\right|}{1+|z|^{N+\lambda}} & \leq C(R, N, \lambda) \int_{B_{\frac{r}{2}(y z)}} \frac{\sup _{B_{r}(y)}\left|u_{0}\right|}{1+|y|^{N+\lambda}} d y \\
& \leq C(R, N, \lambda) \int_{B_{R}(z)} \frac{\sup _{B_{r}(y)}\left|u_{0}\right|}{1+|y|^{N+\lambda}} d y .
\end{aligned}
$$

Let us now integrate wrt $z \in \mathbb{R}^{N}$ :

$$
\begin{aligned}
\left|B_{\frac{r}{2}}\right| \int_{\mathbb{R}^{N}} \sup _{B_{R}(z)}\left|u_{0}\right| \frac{d z}{1+|z|^{N+\lambda}} & \leq C(R, N, \lambda) \int_{\mathbb{R}^{N}} \int_{B_{R}(z)} \frac{\sup _{B_{r}(y)}\left|u_{0}\right|}{1+|y|^{N+\lambda}} d y d z \\
& =C(R, N, \lambda)\left|B_{R}\right| \int_{\mathbb{R}^{N}} \sup _{B_{r}(z)}\left|u_{0}\right| \frac{d z}{1+|z|^{N+\lambda}}
\end{aligned}
$$

the last line is obtained by integrating first wrt z. Since a locally bounded function is integrable wrt $\mu_{b}$ if and only if it is integrable wrt to $\frac{d z}{1+|z|^{N+\lambda}}$, the rest of the proof of the lemma is obvious.

Let us now construct barrier functions that allow us to develop a viscosity solution theory.

Lemma 2.5.2 (Construction of barriers). Let $u_{0}: \mathbb{R}^{N} \rightarrow \mathbb{R}^{+}$be usc and $\mu_{b^{-}}$ uniformly integrable. Then, there exists $\Phi\left[u_{0}\right] \in C^{\infty}\left(\mathbb{R}^{N}\right)$ positive, $\mu_{b}$-uniformly integrable and such that

$$
\begin{aligned}
\forall x \in \mathbb{R}^{N}, u_{0}(x) & \leq \Phi\left[u_{0}\right](x), \\
\lim _{|x| \rightarrow+\infty} \frac{u_{0}(x)}{\Phi\left[u_{0}\right](x)} & =0, \\
\left|\nabla \Phi\left[u_{0}\right]\right|+\left|g\left[\Phi\left[u_{0}\right]\right]\right| & \leq C_{N, \lambda} \Phi\left[u_{0}\right],
\end{aligned}
$$

for some positive constant $C_{N, \lambda}$ that only depends on $N$ and $\lambda$.

Remarks 2.4. (1) We will use this result for usc functions $u: \bar{Q}_{T} \rightarrow \mathbb{R}^{+}$that are $\mu_{b}$-uniformly integrable in space uniformly wrt time. In this case, we apply it to $\sup _{t \in[0, T]} u(t, \cdot)$, and $\Phi[u]$ simply denotes the barrier function $\Phi\left[\sup _{t \in[0, T]} u(t, \cdot)\right]$.

(2) In view of the proof below, one can check that if $u \leq v$, then $\Phi[u] \leq \Phi[v]$.

Proof of Lemma 2.5.2. We can assume without loss of generality that $u_{0} \not \equiv 0$. Define the positive bounded Borel measure

$$
\nu:=\left(\sup _{B_{1}(z)} u_{0}\right) d \mu_{b}(z) .
$$

Consider an increasing sequence $r_{n} \rightarrow+\infty$ such that $r_{n+1} \geq r_{n}+1 / 2$ and

$$
\nu\left(B_{r_{n}}^{c}\right) \leq \frac{1}{n^{3}} \nu\left(\mathbb{R}^{N}\right) .
$$

For $r \geq 0$, define

$$
f(r):=\mathbf{1}_{\left[0, r_{1}+1 / 2\right)}+\sum_{n \geq 1} n \mathbf{1}_{\left[r_{n}+1 / 2, r_{n+1}+1 / 2\right)} .
$$


Notice that $f \geq 1$, is non-decreasing and such that $\lim _{r \rightarrow+\infty} f(r)=+\infty$. Define now for $x \in \mathbb{R}^{N}$,

$$
\Phi(x):=f(|x|)\left(\sup _{B_{\frac{1}{2}}(x)} u_{0}\right) .
$$

Notice that $\Phi$ is $\mu_{b}$-uniformly integrable; indeed,

$$
\begin{aligned}
\int_{\mathbb{R}^{N}}\left(\sup _{B_{\frac{1}{2}}(z)} \Phi\right) d \mu_{b}(z) & \leq \int_{\mathbb{R}^{N}} f(|z|+1 / 2)\left(\sup _{B_{1}(z)} u_{0}\right) d \mu_{b}(z) \\
& =\int_{\mathbb{R}^{N}} f(|z|+1 / 2) d \nu(z) \\
& =\nu\left(B_{r_{1}}\right)+\sum_{n \geq 1} n \nu\left(B_{r_{n+1}} \backslash B_{r_{n}}\right) \\
& \leq \nu\left(B_{r_{1}}\right)+\sum_{n \geq 1} n \nu\left(B_{r_{n}}^{c}\right) \\
& \leq \nu\left(B_{r_{1}}\right)+\sum_{n \geq 1} \frac{n}{n^{3}} \nu\left(\mathbb{R}^{N}\right)<+\infty .
\end{aligned}
$$

Now write $\widetilde{\Phi}:=K(1, \cdot) * \Phi$. This convolution product is finite, thanks to Lemma 2.4.1 which shows that the kernel of $g$ and the density of $\mu$ behaves the same way at infinity (up to a positive multiplicative constant). Moreover, the asymptotic properties of the derivatives of $K$ and the theorem of derivation under the integral sign ensure that $\widetilde{\Phi} \in C^{\infty}\left(\mathbb{R}^{N}\right)$ with $\nabla \widetilde{\Phi}=(\nabla K(1, \cdot)) * \Phi$ and $g[\widetilde{\Phi}]=g[K(1, \cdot)] * \Phi$. Notice that $g[\widetilde{\Phi}]$ is well-defined since we will prove below that $\widetilde{\Phi}$ is $\mu_{b}$-uniformly integrable, thus a fortiori $\mu_{b}$-integrable. We have proved in particular (2.10), since adding to the positivity of $\Phi$, Lemma 2.4.1 also implies that $|\nabla K(1, \cdot)|+|g[K(1, \cdot)]| \leq C_{N, \lambda} K(1, \cdot)$.

Let us now prove that $\widetilde{\Phi}$ is $\mu_{b}$-uniformly integrable. We have

$$
\sup _{B_{1}(\cdot)} \widetilde{\Phi} \leq K(1, \cdot) * \sup _{B_{1}(\cdot)} \Phi
$$

indeed, for $x \in \mathbb{R}^{N}$,

$$
\begin{aligned}
\sup _{B_{1}(x)} \widetilde{\Phi} & =\sup _{z \in B_{1}(x)} \int_{\mathbb{R}^{N}} K(1, y) \Phi(z-y) d y \\
& \leq \int_{\mathbb{R}^{N}} K(1, y) \sup _{z \in B_{1}(x)} \Phi(z-y) d y \\
& =\int_{\mathbb{R}^{N}} K(1, y) \sup _{z^{\prime} \in B_{1}(x-y)} \Phi\left(z^{\prime}\right) d y \\
& =K(1, \cdot) *\left(\sup _{B_{1}(\cdot)} \Phi\right)(x) .
\end{aligned}
$$


Remark that Lemma 2.4 .1 implies that $\mu_{b} \leq C K(1, \cdot)$. Moreover, $K(1, \cdot)$ is even and $K(1, \cdot) \star K(1, \cdot)=K(2, \cdot)$. Hence, it follows that

$$
\begin{aligned}
\int_{\mathbb{R}^{N}} \sup _{B_{1}(z)} \widetilde{\Phi} d \mu_{b}(z) & \leq C \int_{\mathbb{R}^{N}} \sup _{B_{1}(z)} \widetilde{\Phi} K(1,-z) d z \\
& =C K(1, \cdot) * \sup _{B_{1}(\cdot)} \widetilde{\Phi}(0) \\
& \leq C K(1, \cdot) * K(1, \cdot) * \sup _{B_{1}(\cdot)} \Phi(0) \\
& =C K(2, \cdot) * \sup _{B_{1}(\cdot)} \Phi(0) \\
& \leq \widetilde{C} \int_{\mathbb{R}^{N}} \sup _{B_{1}(z)} \Phi d \mu_{b}(z) .
\end{aligned}
$$

The last line is obtained by using the homogeneity property of $K$ which shows that $K(2, \cdot)$ behaves as $K(1, \cdot)$ and a fortiori as the density of $\mu$ at infinity (up to a positive multiplicative constant). Now recalling that $\Phi$ is $\mu_{b}$-uniformly integrable, we deduce that so is $\widetilde{\Phi}$.

We are left to prove (2.8) and (2.9). We have

$$
\widetilde{\Phi}(x) \geq \int_{B_{\frac{1}{2}}(x) \cap\{|z| \geq|x|\}} K(1, x-z) \Phi(z) d z \geq \varepsilon\left(\inf _{z:|z-x| \leq 1 / 2,|z| \geq|x|} \Phi(z)\right),
$$

where $\varepsilon:=\inf _{z \in E_{x}} K(1,-z)$ with $E_{x}:=B_{\frac{1}{2}}(x) \cap\{|z| \geq|x|\}-x$. Since $K(1, \cdot)$ is positive and radial, $\varepsilon$ is a positive constant that does not depend on $x$. On using the definition of $\Phi$, we get

$$
\widetilde{\Phi}(x) \geq \varepsilon \inf _{z:|z-x| \leq 1 / 2,|z| \geq|x|}\left(f(|z|) \sup _{B_{\frac{1}{2}}(z)} u_{0}\right) \geq \varepsilon f(|x|) u_{0}(x) .
$$

One can check that $\Phi\left[u_{0}\right]:=\frac{1}{\varepsilon} \widetilde{\Phi}$ satisfies all the desired properties.

To finish, let us give an example of a non-trivial uniformly integrable function.

Example 2.5.1. Define $e_{1}:=(1,0, \ldots, 0) \in \mathbb{R}^{N}$ and let $x_{1}$ denote the variable on the $e_{1}$-axis of $\mathbb{R}^{N}$. Consider the function defined by

$$
u_{0}(x):=\sum_{n} \mathbf{1}_{B_{1}\left(n^{2} e_{1}\right)}(x)\left|x_{1}\right|^{N-1+\lambda} .
$$

Then $u_{0}$ is uniformly integrable wrt $\mu_{b}$, but clearly does not belong to $C_{\lambda}^{0}$. Moreover, if $N \geq 2$, then $u_{0}$ also does not satisfy the following integrability condition:

$$
\int \sup _{B_{|z|}}\left|u_{0}\right| d \mu_{b}(z)<+\infty
$$

\section{EXIstence AND uniqueness of UnBounded SOlutions For (1.1)}

In this section, we construct a unique unbounded solution of (1.1)-(1.2) for unbounded initial datum $u_{0}$. In order to do so, different assumptions on the behaviour of $u_{0}$ at infinity are made: we will distinguish the cases of sublinear data, superlinear data and "uniformly" $\mu_{b}$ - integrable data. Precisely, we assume that $u_{0}$ satisfies 
one of the following conditions:

$\left|u_{0}(x)\right| \leq C(1+|x|), \quad\left|u_{0}(x)\right| \leq C\left(1+|x|^{\lambda^{\prime}}\right) \quad$ or $\quad \int_{\mathbb{R}^{N}} \sup _{B_{R}(z)}\left|u_{0}(z)\right| d \mu_{b}(z)<+\infty$

for some $C>0, \lambda^{\prime}>1$ and $R>0$. We use the weak notion of viscosity solution in order to get uniqueness, for instance. As mentioned in the Introduction, the assumptions on the non-linearity $H$ vary from one case to the other.

Before treating each case successively, we would like first to discuss the different sets of assumptions we need. To make it clear, let us assume that $H$ is locally Lispchitz continuous, even if this assumption can be relaxed a bit (compare (A2) and $\left(\mathrm{A} 2^{\prime}\right),(\mathrm{B} 2)$ and $\left.\left(\mathrm{B} 2^{\prime}\right)\right)$. As far as the behaviour wrt $u$ is concerned, we classically assume that the Lipschitz constant of $H$ is bounded from below. The way $H$ depends on $(x, p)$ differs from the sublinear case to the superlinear one. If comparison principles are at stake, the Lipschitz constant of $H$ in $x$ has to be sublinear in $p$. But in the superlinear case, this constant can be local in $x$, which is not the case in the sublinear case (compare (A2) and (B2)). Similar differences about the behaviour in $p$ appear (compare (A3) and (B3)). These differences can be understood by looking at the proof arguments: in the sublinear case, the penalization in space parameter vanishes before the doubling variable penalization one, and it is the contrary in the superlinear case. In the latter case, in order to get local bounds for instance, the behaviour of the coefficients must be prescribed (see (B2') and (B4)). If finite propagation speed is expected for the pure Hamilton-Jacobi equation, the Hamiltonian $H$ has to be assumed globally Lipschitz continuous in $p$.

3.1. The sublinear case. We first consider uniformly continuous initial data. This implies that we expect solutions to be sublinear at infinity, i.e. to lie in $C_{1}^{0}$. Naturally, for the range of exponent $\lambda \in(0,1]$, we consider data and solutions that lie more precisely in $C_{\lambda^{\prime}}^{0}$ with $\lambda^{\prime} \in(0, \lambda)$. As far as the non-linearity is concerned, we assume that:

(A1) There exists $\nu \in \mathbb{R}$ such that for all $x \in \mathbb{R}^{N}, u, v \in \mathbb{R}, u \leq v, p \in \mathbb{R}^{N}$, and $t \in[0, T]$

$$
H(t, x, v, p)-H(t, x, u, p) \geq \nu(v-u) .
$$

(A2) There exists a modulus of continuity $m$ such that for all $x, y \in \mathbb{R}^{N}, u \in \mathbb{R}$, $p \in \mathbb{R}^{N}$, and $t \in[0, T]$,

$$
|H(t, x, u, p)-H(t, y, u, p)| \leq m((1+|p|)|x-y|) .
$$

(A3) For all $R>0$, there exists a modulus of continuity $m_{R}$ such that for all $x \in \mathbb{R}^{N}, u \in \mathbb{R}, p, q \in B_{R}$, and $t \in[0, T]$,

$$
|H(t, x, u, p)-H(t, x, u, q)| \leq m_{R}(|p-q|) .
$$

Remark 3.1. It is classical that through a change of unknown function in the equation (namely, $\left.U(t, x):=e^{-(\min \{\nu, 0\}+1) t} u(t, x)\right)$, (A1) reduces to the following assumption:

$\left(\mathrm{A} 1^{\prime}\right)$ For all $x \in \mathbb{R}^{N}, u, v \in \mathbb{R}, u \leq v, p \in \mathbb{R}^{N}$, and $t \in[0, T], H(t, x, v, p)-$ $H(t, x, u, p) \geq v-u$.

For the sake of simplicity, the results of this section are proved under $\left(\mathrm{A} 1^{\prime}\right)$ without changing explicitly the unknown function.

We first obtain a comparison principle. Its proof is given in Appendix A.3. 
Theorem 3.1 (Comparison principle). Assume (A1)-(A3). Let $\lambda^{\prime} \in(0,1] \cap(0, \lambda)$ and $u_{0} \in U C\left(\mathbb{R}^{N}\right) \cap C_{\lambda^{\prime}}^{0}\left(\mathbb{R}^{N}\right)$. Let $u$ and $v$ be, respectively, a sub- and a supersolution of (1.1) such that

$$
\sup _{[0, T] \times \mathbb{R}^{N}} \frac{u^{+}(t, x)}{1+|x|^{\lambda^{\prime}}}<+\infty \quad \text { and } \quad \sup _{[0, T] \times \mathbb{R}^{N}} \frac{v^{-}(t, x)}{1+|x|^{\lambda^{\prime}}}<+\infty .
$$

Then $u \leq v$ whenever $u(0,.) \leq u_{0} \leq v(0,$.$) .$

We now focus on the existence issue and provide an $L_{\mathrm{loc}}^{\infty}$ bound on the solution. We need to prescribe the behaviour at infinity of the source terms of the equations for the range of exponent $\lambda \in(0,1]$ (see Remark 3.2 below).

(A4) There exists a constant $C_{0}$ such that for all $x \in \mathbb{R}^{N}$,

$$
\sup _{[0, T]}|H(t, x, 0,0)| \leq C_{0}\left(1+|x|^{\lambda^{\prime}}\right) .
$$

Theorem 3.2 (Existence and $L_{\text {loc }}^{\infty}$ estimate). Let $\lambda^{\prime} \in(0,1] \cap(0, \lambda)$. Assume (A1)(A4). Let $u_{0} \in U C\left(\mathbb{R}^{N}\right) \cap C_{\lambda^{\prime}}^{0}\left(\mathbb{R}^{N}\right)$. There then exists a (unique) viscosity solution $u \in C_{\lambda^{\prime}}^{0}\left(\bar{Q}_{T}\right)$ of (1.1)-(1.2). Moreover, there exists a constant $M$ only depending on $\left\|u_{0}\right\|_{0, \lambda^{\prime}}$ and $C_{0}$ from (A4) such that $\|u\|_{0, \lambda^{\prime}} \leq M$, i.e. for all $t \in(0, T) \times \mathbb{R}^{N}$

$$
|u(t, x)| \leq M\left(1+|x|^{\lambda^{\prime}}\right) .
$$

Remark 3.2. If $\lambda^{\prime}=1$, then (A2) implies (A4).

In order to get a gradient estimate, assumption (A2) is strenghtened.

$\left(\mathrm{A} 2^{\prime}\right)$ There exists a constant $C_{1} \geq 0$ such that for all $x, y \in \mathbb{R}^{N}, u \in \mathbb{R}, p \in \mathbb{R}^{N}$, and $t \in[0, T]$,

$$
|H(t, x, u, p)-H(t, y, u, p)| \leq C_{1}(1+|p|)|x-y| .
$$

Theorem 3.3 (Gradient estimate). Assume (A1), (A2'), and (A3). Let $\lambda^{\prime} \in$ $(0,1] \cap(0, \lambda)$ and $u_{0} \in C_{\lambda^{\prime}}^{0}\left(\mathbb{R}^{N}\right)$ be Lipschitz continuous. Then, if $u \in C_{\lambda^{\prime}}^{0}\left(\bar{Q}_{T}\right)$ is a viscosity solution of (1.1)-(1.2), then $u$ is Lipschitz continuous wrt the space variable. Moreover there exists a constant $M$, only depending on $\left\|\nabla u_{0}\right\|_{\infty}$ and $C_{1}$ from $\left(\mathrm{A} 2^{\prime}\right)$, such that $\|\nabla u\|_{\infty} \leq M$.

The proofs of the existence and the gradient estimate can easily be adapted from [25] and are left to the reader.

3.2. The superlinear case. We now turn to the superlinear case (1.5). In order to deal with $\lambda>1$ and initial conditions that are not sublinear anymore, the assumptions on the non-linearity $H$ need to be adapted, in particular its regularity wrt the $x$ and $p$ variables. But even in the case $\lambda \leq 1$, we exhibit different conditions on the non-linearity $H$ that ensures the well-posedness of the problem.

(B2) For all $R>0$, there exists a modulus of continuity $m_{R}$ such that for all $x, y \in B_{R}, u \in[-R, R], p \in \mathbb{R}^{N}$, and $t \in[0, T]$,

$$
|H(t, x, u, p)-H(t, y, u, p)| \leq m_{R}((1+|p|)|x-y|) .
$$

(B3) There exists a modulus of continuity $m$ such that for all $x \in \mathbb{R}^{N}, u \in \mathbb{R}$, $p, q \in \mathbb{R}^{N}$, and $t \in[0, T]$,

$$
|H(t, x, u, p)-H(t, x, u, q)| \leq m((1+|x|)|p-q|) .
$$


In this framework, the comparison principle holds true for sub- and supersolutions with (eventual) superlinear growth.

Theorem 3.4 (Comparison principle). Assume (A1), (B2), and (B3). Let $\lambda^{\prime} \in$ $(0, \lambda)$. Let $u$ and $v$ be, respectively, a sub- and a supersolution of (1.1) such that

$$
\sup _{[0, T] \times \mathbb{R}^{N}} \frac{u^{+}(t, x)}{1+|x|^{\lambda^{\prime}}}<+\infty \quad \text { and } \quad \inf _{[0, T] \times \mathbb{R}^{N}} \frac{v^{-}(t, x)}{1+|x|^{\lambda^{\prime}}}>-\infty .
$$

Then $u \leq v$ whenever $u(0,.) \leq v(0,$.$) .$

Proof. Let $M:=\sup _{[0, T] \times \mathbb{R}^{N}}(u-v)$. We must prove that $M \leq 0$. Let us assume the contrary and let us exhibit a contradiction. Let $\lambda^{\prime \prime} \in\left(\lambda^{\prime}, \lambda\right)$ and $\gamma$ be a nonnegative constant that will be appropriately chosen later. Consider two parameters $\varepsilon, \eta>0$ and define

$$
M_{\varepsilon, \eta}=\sup _{[0, T] \times \mathbb{R}^{N} \times \mathbb{R}^{N}}\left(u(t, x)-v(t, y)-\frac{|x-y|^{2}}{2 \varepsilon}-\eta e^{\gamma t} \frac{\left(1+|x|^{2}\right)^{\frac{\lambda^{\prime \prime}}{2}}}{\lambda^{\prime \prime}}\right) .
$$

Since for all $\eta>0$

$$
\lim _{|(x, y)| \rightarrow+\infty} \frac{|x-y|^{2}+\eta\left(1+|x|^{2}\right)^{\frac{\lambda^{\prime \prime}}{2}}}{(1+|x|+|y|)^{\lambda^{\prime}}}=+\infty,
$$

there exists $(\bar{t}, \bar{x}, \bar{y}) \in[0, T] \times \mathbb{R}^{N} \times \mathbb{R}^{N}$ where the supremum is attained. There exists $\eta_{0}>0$ such that for all $\varepsilon>0, M_{\varepsilon, \eta_{0}} \geq M / 2>0$. Moreover,

$$
\begin{aligned}
\lim _{\varepsilon \rightarrow 0} \sup _{\mathbb{R}^{N} \times \mathbb{R}^{N}}(u(0, x)-v(0, y) & \left.-\frac{|x-y|^{2}}{2 \varepsilon}-\eta_{0} \frac{\left(1+|x|^{2}\right)^{\frac{\lambda^{\prime \prime}}{2}}}{\lambda^{\prime \prime}}\right) \\
& =\sup _{\mathbb{R}^{N}}\left(u(0, x)-v(0, x)-\eta_{0} \frac{\left(1+|x|^{2}\right)^{\frac{\lambda^{\prime \prime}}{2}}}{\lambda^{\prime \prime}}\right) \leq 0,
\end{aligned}
$$

and it follows that $\bar{t} \neq 0$ for $\varepsilon$ small enough. By Lemma 2.3.2, there exist

$$
\begin{aligned}
\left(a, p+\eta_{0} e^{\gamma \bar{t}}\left(1+|\bar{x}|^{2}\right)^{\frac{\lambda^{\prime \prime}-2}{2}} \bar{x}\right) & \in \bar{\partial}^{P} u(\bar{t}, \bar{x}), \\
(b, p) & \in \bar{\partial}_{P} v(\bar{t}, \bar{y})
\end{aligned}
$$

with $a-b=\gamma \eta_{0} e^{\gamma \bar{t}} \frac{\left(1+|\bar{x}|^{2}\right)^{\frac{\lambda^{\prime \prime}}{2}}}{\lambda^{\prime \prime}}, p=\frac{\bar{x}-\bar{y}}{\varepsilon}$ and

$$
g[v](\bar{t}, \bar{y}, p)-g[u]\left(\bar{t}, \bar{x}, p+\eta_{0} e^{\gamma \bar{t}}\left(1+|\bar{x}|^{2}\right)^{\frac{\lambda^{\prime \prime}-2}{2}} \bar{x}\right) \leq-\frac{\eta_{0} e^{\gamma \bar{t}}}{\lambda^{\prime \prime}} g\left[\left(1+|\cdot|^{2}\right)^{\frac{\lambda^{\prime \prime}}{2}}\right](\bar{x}) .
$$

Subtracting the corresponding viscosity inequalities and using ( $\left.\mathrm{A} 1^{\prime}\right)$ (see Remark 3.1), we get

$$
\begin{aligned}
M / 2 & +\gamma \eta_{0} \frac{e^{\gamma \bar{t}}\left(1+|\bar{x}|^{2}\right)^{\frac{\lambda^{\prime \prime}}{2}}}{\lambda^{\prime \prime}} \leq H(\bar{t}, \bar{y}, u(\bar{t}, \bar{x}), p) \\
& -H\left(\bar{t}, \bar{x}, u(\bar{t}, \bar{x}), p+\eta_{0} e^{\gamma \bar{t}}\left(1+|\bar{x}|^{2}\right)^{\frac{\lambda^{\prime \prime}-2}{2}} \bar{x}\right)-\frac{\eta_{0} e^{\gamma \bar{t}}}{\lambda^{\prime \prime}} g\left[\left(1+|\cdot|^{2}\right)^{\frac{\lambda^{\prime \prime}}{2}}\right](\bar{x}) .
\end{aligned}
$$


By (3.1), there exists $R \geq 0$, that depends on $\eta_{0}$ but not on $0<\varepsilon \leq 1$, such that $\bar{x}, \bar{y} \in B_{R}$ and $u(\bar{t}, \bar{x}) \in[-R, R]$. By (B2) and (B3), we get

$$
\begin{aligned}
M / 2+\gamma \eta_{0} \frac{e^{\gamma \bar{t}}\left(1+|\bar{x}|^{2}\right)^{\frac{\lambda^{\prime \prime}}{2}}}{\lambda^{\prime \prime}} & \leq m_{R}\left(|\bar{x}-\bar{y}|+\frac{|\bar{x}-\bar{y}|^{2}}{\varepsilon}\right) \\
+ & m\left(2 \eta_{0} e^{\gamma \bar{t}}\left(1+|\bar{x}|^{2}\right)^{\frac{\lambda^{\prime \prime}}{2}}\right)-\frac{\eta_{0} e^{\gamma \bar{t}}}{\lambda^{\prime \prime}} g\left[\left(1+|\cdot|^{2}\right)^{\frac{\lambda^{\prime \prime}}{2}}\right](\bar{x}) .
\end{aligned}
$$

By Lemma B.0.1 in Appendix B, the function $g\left[\left(1+|\cdot|^{2}\right)^{\frac{\lambda^{\prime \prime}}{2}}\right]$ is bounded. If $C$ denotes its $L^{\infty}$ norm and $\gamma=C+2 \lambda^{\prime \prime} K$ (where $K$ is an arbitrary positive real number), then

$$
\begin{aligned}
M / 2 & \leq m_{R}\left(|\bar{x}-\bar{y}|+\frac{|\bar{x}-\bar{y}|^{2}}{\varepsilon}\right)+m\left(2 \eta_{0} e^{\gamma \bar{t}}\left(1+|\bar{x}|^{2}\right)^{\frac{\lambda^{\prime \prime}}{2}}\right)-2 K \eta_{0} e^{\gamma \bar{t}}\left(1+|\bar{x}|^{2}\right)^{\frac{\lambda^{\prime \prime}}{2}} \\
& \leq m_{R}\left(|\bar{x}-\bar{y}|+\frac{|\bar{x}-\bar{y}|^{2}}{\varepsilon}\right)+\sup _{r>0}(m(r)-K r) .
\end{aligned}
$$

Classical arguments allow us to prove that $\lim _{\varepsilon \rightarrow 0} \frac{|\bar{x}-\bar{y}|^{2}}{\varepsilon}=0$, since $\eta_{0}$ is a fixed positive number. The limit as $\varepsilon \rightarrow 0$ thus yields $M / 2 \leq \sup _{r>0}(m(r)-K r)$. Taking the infimum wrt $K>0$ gives the contradiction $0<M / 2 \leq 0$. The proof of Theorem 3.4 is now complete.

Theorem 3.5 (Existence and $L_{\text {loc }}^{\infty}$ estimate). Let $\lambda^{\prime} \in(0, \lambda)$. Assume (A1), (B2), (B3), and (A4). Let $u_{0} \in C_{\lambda^{\prime}}^{0}\left(\mathbb{R}^{N}\right)$. There then exists a (unique) viscosity solution $u \in C_{\lambda^{\prime}}^{0}\left(\bar{Q}_{T}\right)$ of (1.1)-(1.2). Moreover, $\|u\|_{0, \lambda^{\prime}} \leq M$, i.e. for all $(t, x) \in(0, T) \times \mathbb{R}^{N}$

$$
|u(t, x)| \leq M\left(1+|x|^{\lambda^{\prime}}\right)
$$

where $M$ is some constant that only depends on $\left\|u_{0}\right\|_{0, \lambda^{\prime}}, C_{0}$ from (A4) and $m$ from (B3).

We do not give an explicit proof of this result, since it is similar and easier than the one we will give for the existence of solutions with integrable initial data (see the next subsection).

We now turn to gradient estimates. To get some, we need to make precise the way the derivative of the non-linearity $H$ wrt $x$ depends on $|x|$ (see $R$ in (B2)) and to strengthen (B3) a little bit.

(B2') There exists a constant $C_{1}$ such that for all $x, y \in \mathbb{R}^{N}, u \in \mathbb{R}, p \in \mathbb{R}^{N}$, and $t \in[0, T]$

$$
|H(t, x, u, p)-H(t, y, u, p)| \leq C_{1}\left(1+|x|^{\lambda^{\prime}-1}+|y|^{\lambda^{\prime}-1}+|p|\right)|x-y| .
$$

$\left(\mathrm{B}^{\prime}\right)$ There exists a constant $C_{2}$ such that for all $x, y \in \mathbb{R}^{N}, u \in \mathbb{R}, p, q \in \mathbb{R}^{N}$, and $t \in[0, T]$,

$$
|H(t, x, u, p)-H(t, x, u, q)| \leq C_{2}(1+|x|)|p-q| .
$$

Theorem 3.6 (Gradient estimate). Let $\lambda \in(1,2)$ and $\lambda^{\prime} \in(1, \lambda)$. Assume (A1), $\left(\mathrm{B} 2^{\prime}\right)$, and $\left(\mathrm{B} 3^{\prime}\right)$. Let $u_{0} \in C_{\lambda^{\prime}}^{0,1}\left(\mathbb{R}^{N}\right)$. If $u \in C_{\lambda^{\prime}}^{0}\left(\bar{Q}_{T}\right)$ is a viscosity solution of (1.1) -(1.2), then $u \in C_{\lambda^{\prime}}^{0,1}\left(\bar{Q}_{T}\right)$ and there exists a constant $M$, only depending on $\left\|u_{0}\right\|_{1, \lambda^{\prime}}, C_{1}$ from $\left(\mathrm{B}^{\prime}\right)$ and $C_{2}$ from $\left(\mathrm{B}^{\prime}\right)$, such that $\|u\|_{1, \lambda^{\prime}} \leq M$. 
Proof. Consider a parameter $\varepsilon>0$ and define

$$
u^{\varepsilon}(t, x):=\sup _{y \in \mathbb{R}^{N}}\left(u(t, y)-e^{\gamma t} \frac{|x-y|^{2}}{2 \varepsilon}\right)
$$

with

$$
\gamma=\max \left\{2 C_{1}+1,4 C_{2}\left(\lambda^{\prime}-1\right)+\left\|g\left[\left(1+|\cdot|^{2}\right)^{\lambda^{\prime}-1}\right]\right\|_{\infty}+1\right\},
$$

where $C_{1}$ and $C_{2}$ appear in $\left(\mathrm{B}^{\prime}\right)$ and $\left(\mathrm{B} 3^{\prime}\right)$. It is easy to see that $u^{\varepsilon}$ is continuous and such that $\sup _{[0, T] \times \mathbb{R}^{N}} \frac{u^{\varepsilon}(t, x)}{1+|x|^{\lambda^{\prime}}}<+\infty$. Let us prove that $u^{\varepsilon}$ is a viscosity subsolution of the following equation:

$$
\partial_{t} u^{\varepsilon}+H\left(t, x, u^{\varepsilon}, \nabla u^{\varepsilon}\right)+g\left[u^{\varepsilon}\right] \leq C \varepsilon \frac{\left(1+|x|^{2}\right)^{\lambda^{\prime}-1}}{2\left(\lambda^{\prime}-1\right)}+C \varepsilon^{\frac{\lambda^{\prime}}{2-\lambda^{\prime}}},
$$

where $C$ is some constant that only depends on $C_{1}$ and $\lambda^{\prime}$. Let $t \in(0, T], x \in \mathbb{R}^{N}$ and $(a, p) \in \partial^{P} u^{\varepsilon}(t, x)$. It is well-known that there exists a point $\bar{y}$ such that $u^{\varepsilon}(t, x)=u(t, \bar{y})-e^{\gamma t} \frac{|x-\bar{y}|^{2}}{2 \varepsilon},\left(a+\gamma e^{\gamma t} \frac{|x-\bar{y}|^{2}}{2 \varepsilon}, p\right) \in \partial^{P} u(t, \bar{y})$ and $p=e^{\gamma t}\left(\frac{x-\bar{y}}{\varepsilon}\right)$. Moreover, using the fact that

$$
u^{\varepsilon}(t, x+z)-u^{\varepsilon}(t, x)-\frac{p \cdot z}{1+|z|^{2}} \geq u(t, \bar{y}+z)-u(t, \bar{y})-\frac{p \cdot z}{1+|z|^{2}},
$$

we get $g\left[u^{\varepsilon}\right](t, x, p) \leq g[u](t, \bar{y}, p)$. The viscosity inequality corresponding to the solution $u$ of (1.1) at $(t, \bar{y})$ and assumptions $\left(\mathrm{A}^{\prime}\right)$ (see Remark 3.1) and (B2') then imply that

$$
\begin{aligned}
a+H(t, x, & \left.u^{\varepsilon}(t, x), p\right)+g\left[u^{\varepsilon}\right](t, x, p) \\
& \leq C_{1}\left(\left(1+|x|^{\lambda^{\prime}-1}+|\bar{y}|^{\lambda^{\prime}-1}\right)+e^{\gamma t} \frac{|x-\bar{y}|}{\varepsilon}\right)|x-\bar{y}|-\gamma e^{\gamma t} \frac{|x-\bar{y}|^{2}}{2 \varepsilon} .
\end{aligned}
$$

By (3.3), the right-hand side of this inequality is bounded from above by

$$
\begin{aligned}
C_{1}(1+ & \left.2|x|^{\lambda^{\prime}-1}\right)|x-\bar{y}|+C_{1}|x-\bar{y}|^{\lambda^{\prime}}+C_{1} e^{\gamma t} \frac{|x-\bar{y}|^{2}}{\varepsilon}-\gamma e^{\gamma t} \frac{|x-\bar{y}|^{2}}{2 \varepsilon} \\
& \leq \sup _{r>0}\left(C_{1}\left(1+2|x|^{\lambda^{\prime}-1}\right) r+C_{1} r^{\lambda^{\prime}}-\frac{r^{2}}{2 \varepsilon}\right) \leq C \frac{\left(1+|x|^{2}\right)^{\lambda^{\prime}-1}}{2\left(\lambda^{\prime}-1\right)} \varepsilon+C \varepsilon^{\frac{\lambda^{\prime}}{2-\lambda^{\prime}}},
\end{aligned}
$$

where $C$ is some constant that only depends on $C_{1}$ and $\lambda^{\prime}$. This achieves the proof of (3.4).

Let us prove that for all $C^{\prime} \geq C$,

$$
w(t, x):=u^{\varepsilon}(t, x)-C^{\prime} e^{\gamma t} \varepsilon \frac{\left(1+|x|^{2}\right)^{\lambda^{\prime}-1}}{2\left(\lambda^{\prime}-1\right)}-C \varepsilon^{\frac{\lambda^{\prime}}{2-\lambda^{\prime}}} t
$$

is a subsolution of (1.1). Let $t \in(0, T], x \in \mathbb{R}^{N}$ and $(a, p) \in \partial^{P} w(t, x)$. Then,

$$
\left(a+\gamma C^{\prime} e^{\gamma t} \varepsilon \frac{\left(1+|x|^{2}\right)^{\lambda^{\prime}-1}}{2\left(\lambda^{\prime}-1\right)}+C \varepsilon^{\frac{\lambda^{\prime}}{2-\lambda^{\prime}}}, p+C^{\prime} e^{\gamma t} \varepsilon\left(1+|x|^{2}\right)^{\lambda^{\prime}-2} x\right) \in \partial^{P} u^{\varepsilon}(t, x)
$$

and

$$
\begin{aligned}
g[w](t, x, p)=g\left[u^{\varepsilon}\right]\left(t, x, p+C^{\prime} e^{\gamma t} \varepsilon\left(1+|x|^{2}\right)^{\lambda^{\prime}-2} x\right) \\
-C^{\prime} e^{\gamma t} \varepsilon \frac{1}{2\left(\lambda^{\prime}-1\right)} g\left[\left(1+|\cdot|^{2}\right)^{\lambda^{\prime}-1}\right](x) .
\end{aligned}
$$


The viscosity inequality corresponding to the subsolution $u^{\varepsilon}$ of (3.4) and assumptions $\left(\mathrm{A} 1^{\prime}\right)$ and $\left(\mathrm{B} 3^{\prime}\right)$ then imply that

$$
\begin{aligned}
& a+H(t, x, w(t, x), p)+g[w](t, x, p) \leq 2 C_{2} C^{\prime} e^{\gamma t} \varepsilon\left(1+|x|^{2}\right)^{\lambda^{\prime}-1} \\
+ & C \frac{\left(1+|x|^{2}\right)^{\lambda^{\prime}-1}}{2\left(\lambda^{\prime}-1\right)} \varepsilon-C^{\prime} e^{\gamma t} \varepsilon \frac{1}{2\left(\lambda^{\prime}-1\right)} g\left[\left(1+|\cdot|^{2}\right)^{\lambda^{\prime}-1}\right](x)-\gamma C^{\prime} e^{\gamma t} \varepsilon \frac{\left(1+|x|^{2}\right)^{\lambda^{\prime}-1}}{2\left(\lambda^{\prime}-1\right)} .
\end{aligned}
$$

By (3.3), the right-hand side of this inequality is non-positive for $C^{\prime} \geq C$, and we conclude that $w$ is a subsolution of (1.1).

Clearly $\sup _{\bar{Q}_{T}} \frac{w(t, x)}{1+|x|^{\lambda^{\prime}}}<+\infty$, and by the comparison principle we get

$$
w(t, x) \leq u(t, x)+\sup _{\mathbb{R}^{N}}\left(w(0, x)-u_{0}(x)\right)
$$

for all $t \in[0, T]$ and all $x \in \mathbb{R}^{N}$. Simple computations show that

$$
\begin{gathered}
\sup _{\mathbb{R}^{N}}\left(w(0, x)-u_{0}(x)\right)=\sup _{\mathbb{R}^{N} \times \mathbb{R}^{N}}\left(u_{0}(y)-u_{0}(x)-\frac{|x-y|^{2}}{2 \varepsilon}-C^{\prime} \varepsilon \frac{\left(1+|x|^{2}\right)^{\lambda^{\prime}-1}}{2\left(\lambda^{\prime}-1\right)}\right) \\
\leq \sup _{\mathbb{R}^{N} \times \mathbb{R}^{N}}\left(\left\|u_{0}\right\|_{1, \lambda^{\prime}}\left(1+|x|^{\lambda^{\prime}-1}+|y|^{\lambda^{\prime}-1}\right)|x-y|-\frac{|x-y|^{2}}{2 \varepsilon}-C^{\prime} \varepsilon \frac{\left(1+|x|^{2}\right)^{\lambda^{\prime}-1}}{2\left(\lambda^{\prime}-1\right)}\right) \\
\leq \sup _{\mathbb{R}^{N} \times \mathbb{R}^{N}}\left(\left\|u_{0}\right\|_{1, \lambda^{\prime}}\left(1+2|x|^{\lambda^{\prime}-1}\right)|x-y|+\left\|u_{0}\right\|_{1, \lambda^{\prime}}|x-y|^{\lambda^{\prime}}\right. \\
\left.-\frac{|x-y|^{2}}{2 \varepsilon}-C^{\prime} \varepsilon \frac{\left(1+|x|^{2}\right)^{\lambda^{\prime}-1}}{2\left(\lambda^{\prime}-1\right)}\right) \\
\leq \sup _{x \in \mathbb{R}^{N}}\left(\sup _{r>0}\left(\left\|u_{0}\right\|_{1, \lambda^{\prime}}\left(1+2|x|^{\lambda^{\prime}-1}\right) r+\left\|u_{0}\right\|_{1, \lambda^{\prime}} r^{\lambda^{\prime}}-\frac{r^{2}}{2 \varepsilon}\right)-C^{\prime} \varepsilon \frac{\left(1+|x|^{2}\right)^{\lambda^{\prime}-1}}{2\left(\lambda^{\prime}-1\right)}\right) \\
\leq \sup _{x \in \mathbb{R}^{N}}\left(C^{\prime \prime} \varepsilon \frac{\left(1+|x|^{2}\right)^{\lambda^{\prime}-1}}{2\left(\lambda^{\prime}-1\right)}+C^{\prime \prime} \varepsilon^{\frac{\lambda^{\prime}-\lambda^{\prime}}{2-1}}-C^{\prime} \varepsilon \frac{\left(1+|x|^{2}\right)^{\lambda^{\prime}-1}}{2\left(\lambda^{\prime}-1\right)}\right),
\end{gathered}
$$

where $C^{\prime \prime}$ only depends on $\left\|u_{0}\right\|_{1, \lambda^{\prime}}$ and $\lambda^{\prime}$. If we take $C^{\prime} \geq C^{\prime \prime}$ sufficiently large, then

$$
\sup _{\mathbb{R}^{N}}\left(w(0, x)-u_{0}(x)\right) \leq C^{\prime \prime} \varepsilon^{\frac{\lambda^{\prime}}{2-\lambda^{\prime}}} .
$$

Inequality (3.5) then implies that for all $t \in[0, T], x, y \in \mathbb{R}^{N}$ and $\varepsilon>0$,

$$
u(t, y) \leq u(t, x)+e^{\gamma t} \frac{|x-y|^{2}}{2 \varepsilon}+C^{\prime} e^{\gamma t} \varepsilon \frac{\left(1+|x|^{2}\right)^{\lambda^{\prime}-1}}{2\left(\lambda^{\prime}-1\right)}+\varepsilon^{\frac{\lambda^{\prime}}{2-\lambda}}\left(C^{\prime \prime}+C t\right) .
$$

It follows that

$$
|u(t, x)-u(t, y)| \leq e^{\gamma t} \frac{|x-y|^{2}}{2 \varepsilon}+C^{\prime} e^{\gamma t} \varepsilon \frac{\left(1+|x|^{2}\right)^{\lambda^{\prime}-1}}{2\left(\lambda^{\prime}-1\right)}+\varepsilon^{\frac{\lambda^{\prime}}{2-\lambda^{\prime}}}\left(C^{\prime \prime}+C t\right),
$$

and taking $\varepsilon=\frac{|x-y|}{\left(1+|x|^{\lambda^{\prime}-1}\right)}$ implies the result. 
3.3. The uniformly integrable framework. In this subsection we present a viscosity solution theory in a uniformly integrable framework (see the definition in Section 21). As we shall see, this theory seems to be related to a property of the speed of propagation for (1.1). It is as if the equation keeps memory of the finite propagation speed of the associated pure first order Hamilton-Jacobi equation (i.e. (1.1) without the integral term).

Let us begin by proving a strong uniqueness result. To do this, assumption (B3) is strengthened as follows:

(C3) There exists a modulus of continuity such that for all $x, y \in \mathbb{R}^{N}, p, q \in \mathbb{R}^{N}$, $u \in \mathbb{R}$, and $t \in[0, T]$,

$$
|H(t, x, u, p)-H(t, x, u, q)| \leq m(|p-q|) .
$$

Theorem 3.7 (Comparison principle). Assume (A1), (B2), and (C3). Let $u$ and $v$ be, respectively, a sub- and a supersolution of (1.1) such that $u^{+}$and $v^{-}$are $\mu_{b^{-}}$ uniformly integrable in space uniformly wrt time. Then $u \leq v$ whenever $u(0,$. $v(0,$.$) .$

Proof. Let us assume as before that $M:=\sup _{[0, T] \times \mathbb{R}^{N}}(u-v)>0$ and let us exhibit a contradiction. We need to construct barrier functions in order to perturb this supremum. We do so by applying Lemma 2.5.2. Precisely, define $\Phi:=\Phi\left[u^{+}\right]+\Phi\left[v^{-}\right]$ and

$$
M_{\varepsilon, \eta}=\sup _{[0, T] \times \mathbb{R}^{N} \times \mathbb{R}^{N}}\left(u(t, x)-v(t, y)-\frac{|x-y|^{2}}{2 \varepsilon}-\eta e^{\gamma t}(\Phi(x)+\Phi(y))\right) .
$$

By property (2.9) of barrier functions, we claim that there exists $(\bar{t}, \bar{x}, \bar{y}) \in[0, T] \times$ $\mathbb{R}^{N} \times \mathbb{R}^{N}$, where the supremum is attained. Arguing as in the proof of Theorem 3.4. we infer that $M_{\varepsilon, \eta_{0}} \geq M / 2$ and $\bar{t} \neq 0$ for some $\eta_{0}>0$ and all $\varepsilon$ small enough. Lemma 2.3.2 yields the existence of

$$
\begin{aligned}
& \left(a, p+\eta_{0} e^{\gamma \bar{t}} \nabla \Phi(\bar{x})\right) \in \bar{\partial}^{P} u(\bar{t}, \bar{x}), \\
& \left(b, p-\eta_{0} e^{\gamma \bar{t}} \nabla \Phi(\bar{y})\right) \in \bar{\partial}_{P} v(\bar{t}, \bar{y})
\end{aligned}
$$

with $a-b=\gamma \eta_{0} e^{\gamma \bar{t}}(\Phi(\bar{x})+\Phi(\bar{y})), p=\frac{\bar{x}-\bar{y}}{\varepsilon}$ and

$$
\begin{aligned}
& g[v]\left(\bar{t}, \bar{y}, p-\eta_{0} e^{\gamma \bar{t}} \nabla \Phi(\bar{y})\right)-g[u]\left(\bar{t}, \bar{x}, p+\eta_{0} e^{\gamma \bar{t}} \nabla \Phi(\bar{x})\right) \\
& \leq-\eta_{0} e^{\gamma \bar{t}}(g[\Phi](\bar{x})+g[\Phi](\bar{y})) .
\end{aligned}
$$

The viscosity inequalities and $\left(\mathrm{A} 1^{\prime}\right)$ now give

$$
\begin{aligned}
M / 2+\gamma \eta_{0} e^{\gamma \bar{t}} & (\Phi(\bar{x})+\Phi(\bar{y})) \leq H\left(\bar{t}, \bar{y}, u(\bar{t}, \bar{x}), p-\eta_{0} e^{\gamma \bar{t}} \nabla \Phi(\bar{y})\right) \\
& -H\left(\bar{t}, \bar{x}, u(\bar{t}, \bar{x}), p+\eta_{0} e^{\gamma \bar{t}} \nabla \Phi(\bar{x})\right)-\eta_{0} e^{\gamma \bar{t}}(g[\Phi](\bar{x})+g[\Phi](\bar{y}))
\end{aligned}
$$

Again using property (2.9) of the barrier functions, we claim that $\bar{x}, \bar{y} \in B_{R}$ and $u(\bar{t}, \bar{x}) \in[-R, R]$ for some $R \geq 0$ which does not depend on $0<\varepsilon \leq 1$ (but does 
depend on $\left.\eta_{0}\right)$. By (B2) and (C3), we get

$$
\begin{aligned}
M / 2+\gamma \eta_{0} e^{\gamma \bar{t}} & (\Phi(\bar{x})+\Phi(\bar{y})) \leq m_{R}\left(|\bar{x}-\bar{y}|+\frac{|\bar{x}-\bar{y}|^{2}}{\varepsilon}\right) \\
& +m\left(\eta_{0} e^{\gamma \bar{t}}(|\nabla \Phi(\bar{x})|+|\nabla \Phi(\bar{y})|)\right)-\eta_{0} e^{\gamma \bar{t}}(g[\Phi](\bar{x})+g[\Phi](\bar{y})) .
\end{aligned}
$$

We next use the key property on the barrier function, (2.10), to get

$$
\begin{aligned}
|\nabla \Phi|+g[\Phi] & \leq\left|\nabla \Phi\left[u^{+}\right]\right|+g\left[\Phi\left[u^{+}\right]\right]+\left|\nabla \Phi\left[v^{-}\right]\right|+g\left[\Phi\left[v^{-}\right]\right] \\
& \leq C_{N, \lambda}\left(\Phi\left[u^{+}\right]+\Phi\left[v^{-}\right]\right)=C_{N, \lambda} \Phi .
\end{aligned}
$$

Combining the two previous inequalities yields

$$
\begin{aligned}
M / 2+\gamma \eta_{0} e^{\gamma \bar{t}}( & \Phi(\bar{x})+\Phi(\bar{y})) \leq m_{R}\left(|\bar{x}-\bar{y}|+\frac{|\bar{x}-\bar{y}|^{2}}{\varepsilon}\right) \\
& +m\left(C_{N, \lambda} \eta_{0} e^{\gamma \bar{t}}(\Phi(\bar{x})+\Phi(\bar{y}))\right)+C_{N, \lambda} \eta_{0} e^{\gamma \bar{t}}(\Phi(\bar{x})+\Phi(\bar{y})),
\end{aligned}
$$

and, on taking $\gamma=C_{N, \lambda}(K+1)$, we deduce that

$$
M / 2 \leq m_{R}\left(|\bar{x}-\bar{y}|+\frac{|\bar{x}-\bar{y}|^{2}}{\varepsilon}\right)+\sup _{r>0}(m(r)-K r) .
$$

Now taking successively the limit as $\varepsilon \rightarrow 0$ and the infimum wrt $K>0$ we get the desired contradiction $0<M / 2 \leq 0$. The proof is complete.

To get the existence result, we naturally need a uniform integrability condition on source terms:

(C4) The function $(t, x) \mapsto H(t, x, 0,0)$ is $\mu_{b}$-uniformly integrable in space uniformly wrt time.

Theorem 3.8 (Existence and $L_{\text {loc }}^{\infty}$ estimate). Assume (A1), (B2), (C3), and (C4). Let $u_{0}$ be continuous and $\mu_{b}$-uniformly integrable. Then there exists a (unique) continuous and $\mu_{b}$-uniformly integrable in space (uniformly wrt time) viscosity solution $u$ of (1.1) -(1.2).

Proof. Let us first approximate the initial condition $u_{0}$ and the Hamiltonian $H$ in order to apply the result of [25]. Define $u_{n}^{0}:=u_{0} \times \chi_{n}$, where $\chi_{n}: \mathbb{R}^{N} \rightarrow$ $[0,1]$ equals 1 in $B_{n}$ and 0 outside $B_{n+1}$. We also define $H_{n}:=T_{n}(H)$, where $T_{n}(r):=\max (-n, \min (n, r))$ is a truncature function. The theory of [25] ensures the existence of a bounded continuous viscosity solution $u_{n}$ associated with these data. Assume for a while that we have some $L_{l o c}^{\infty}$ estimate on $u_{n}$. Proposition 2.1 implies that the upper (resp. lower) semi-limit of $u_{n}$ is a subsolution (resp. supersolution) of (1.1)-(1.2). The comparison principle then yields limsup* $u_{n} \leq \liminf { }_{*} u_{n}$. Since the other inequality always holds true, we have $\lim \sup ^{*} u_{n}=\liminf { }_{*} u_{n}$. If $u$ denotes this function, we see that $u$ is both usc and lsc and both a viscosity suband supersolution of (1.1)-(1.2).

It now remains to derive an $L_{l o c}^{\infty}$ estimate on $u_{n}$. To do this, define $\Phi:=\Phi\left[\left|u_{0}\right|\right]+$ $\Phi[|H(\cdot, \cdot, 0,0)|]$. In view of Remarks 2.4 following Lemma 2.5.2. we claim that $\Phi \geq$ $\Phi\left[\left|u_{n}^{0}\right|\right]+\Phi\left[\left|H_{n}(\cdot, \cdot, 0,0)\right|\right]$. Hence, we have

$$
\begin{aligned}
\left|u_{n}^{0}\right|+\sup _{[0, T]}\left|H_{n}(t, \cdot, 0,0)\right| & \leq \Phi, \\
|\nabla \Phi|+|g[\Phi]| & \leq C_{N, \lambda} \Phi .
\end{aligned}
$$


Let us look for a supersolution of (1.1) of the form

$$
w(t, x):=e^{\gamma t} \Phi(x)+t \sup _{r>0}(m(r)-K r) .
$$

We first choose $K$ sufficiently large so that $\sup _{r>0}(m(r)-K r)$ is finite. By using $\left(\mathrm{A} 1^{\prime}\right)$ and $(\mathrm{C} 3)$, simple computations show that we have

$$
\begin{aligned}
\partial_{t} w(t, x)+H_{n}(t, x, w, \nabla w)+ & g[w] \geq \gamma e^{\gamma t} \Phi(x)+\sup _{r>0}(m(r)-K r) \\
& +H_{n}(t, x, 0,0)-m\left(e^{\gamma t}|\nabla \Phi(x)|\right)+e^{\gamma t} g[\Phi](x) .
\end{aligned}
$$

By the property on the barrier function, we see that

$$
\begin{aligned}
\partial_{t} w(t, x)+H_{n}(t, x, w, \nabla w)+g[w] \geq \gamma e^{\gamma t} \Phi(x)+\sup _{r>0}(m(r)-K r) \\
\\
\quad-e^{\gamma t} \Phi(x)-m\left(C_{N, \lambda} e^{\gamma t} \Phi(x)\right)-C_{N, \lambda} e^{\gamma t} \Phi(x) .
\end{aligned}
$$

If we take $\gamma:=1+C_{N, \lambda}(1+K)$, we get

$$
\partial_{t} w(t, x)+H_{n}(t, x, w, \nabla w)+g[w] \geq 0 .
$$

The comparison principle then implies that $u_{n} \leq w$. We argue similarly to get the other inequality and prove that $\left|u_{n}\right| \leq w$. This is the desired $L_{l o c}^{\infty}$ estimate, and the proof is now complete.

To get a Lipschitz continuity regularity result, we need to use the following assumptions:

(C2) The Hamiltonian $H$ is locally Lipschitz continuous wrt $x$, and there are a constant $C_{1}$ and a locally bounded function $\Theta: \mathbb{R}^{N} \rightarrow \mathbb{R}^{+}$such that $\Theta^{2}$ is $\mu_{b}$-uniformly integrable and for a.e. $x \in \mathbb{R}^{N}, u \in \mathbb{R}, p \in \mathbb{R}^{N}$, and $t \in[0, T]$,

$$
\left|\nabla_{x} H(t, x, u, p)\right| \leq \Theta(x)+C_{1}|p| .
$$

(C3') There exists a constant $C_{2}$ such that for all $x, y \in \mathbb{R}^{N}, u \in \mathbb{R}, p, q \in \mathbb{R}^{N}$, and $t \in[0, T]$

$$
|H(t, x, u, p)-H(t, x, u, q)| \leq C_{2}|p-q| .
$$

Theorem 3.9 (Gradient estimate). Assume (A1), (C2), and (C3'). Let $u_{0}$ be locally Lipschitz continuous, $\mu_{b}$-uniformly integrable and such that $\left|\nabla u_{0}\right|^{2}$ is $\mu_{b^{-}}$ uniformly integrable. Then, if $u$ is a continuous viscosity solution of (1.1)-(1.2) $\mu_{b}$-uniformly integrable in space uniformly wrt time, then $u$ is locally Lipschitz continuous wrt the space variable.

Proof. Consider $\Phi:=\Phi[|u|]+\Phi\left[\left|\nabla u_{0}\right|^{2}\right]+\Phi\left[\Theta^{2}\right]$ a barrier function given by Lemma 2.5.2. For $x, y \in \mathbb{R}^{N}$, define

$$
\phi(x, y):=\int_{0}^{1} \Phi((1-\tau) x+\tau y) d \tau+\Phi(x)+\Phi(y) .
$$

For $\varepsilon>0$, define

$$
M:=\sup _{[0, T] \times \mathbb{R}^{N} \times \mathbb{R}^{N}}\left(u(t, x)-u(t, y)-e^{\gamma t} \frac{|x-y|^{2}}{2 \varepsilon}-\varepsilon e^{\gamma t} \phi(x, y)\right) .
$$

Let us prove that for $\gamma$ sufficiently large, this supremum is non-positive for all $\varepsilon>0$. Assume this is true. In this case, for every $x, y \in \mathbb{R}^{N}$ and $\varepsilon>0$, we have

$$
u(t, y)-u(t, x) \leq e^{\gamma t} \frac{|x-y|^{2}}{2 \varepsilon}+\varepsilon e^{\gamma t} \phi(x, y) .
$$


It follows that

$$
|u(t, x)-u(t, y)| \leq e^{\gamma t} \frac{|x-y|^{2}}{2 \varepsilon}+\varepsilon e^{\gamma t} \phi(x, y),
$$

and taking $\varepsilon=\frac{|x-y|}{\phi(x, y)}$ implies the result since $\phi$ is locally bounded.

It remains to prove that $M$ is non-positive. Recall that $M$ is achieved at some $(\bar{t}, \bar{x}, \bar{y}) \in[0, T] \times \mathbb{R}^{N} \times \mathbb{R}^{N}$, thanks to the definition of $\phi$ and to property (2.9) of $\Phi[|u|]$. If $\bar{t}=0$, then

$$
M \leq \sup _{\mathbb{R}^{N} \times \mathbb{R}^{N}}\left(u_{0}(x)-u_{0}(y)-\frac{|x-y|^{2}}{2 \varepsilon}-\varepsilon \phi(x, y)\right) .
$$

Using property (2.8) of the barrier function, we claim that $\Phi$ is continuous and such that $\Phi \geq\left|\nabla u_{0}\right|^{2}$. Hence, it is obvious that for every $x, y \in \mathbb{R}^{N}$,

$$
\left|u_{0}(x)-u_{0}(y)\right| \leq I(x, y)|x-y|,
$$

where $I(x, y):=\int_{0}^{1} \sqrt{\Phi((1-\tau) x+\tau y)} d \tau$. It follows that

$$
M \leq \sup _{\mathbb{R}^{N} \times \mathbb{R}^{N}}\left(I(x, y)|x-y|-\frac{|x-y|^{2}}{2 \varepsilon}-\varepsilon \phi(x, y)\right) .
$$

But, $I(x, y)|x-y|-\frac{|x-y|^{2}}{2 \varepsilon} \leq \sup _{r>0}\left(I(x, y) r-\frac{r^{2}}{2 \varepsilon}\right)$, and an easy computation shows that this supremum equals $\frac{1}{2} I^{2}(x, y) \varepsilon$. Since Jensen's inequality implies that

$$
I^{2}(x, y)=\left(\int_{0}^{1} \sqrt{\Phi((1-\tau) x+\tau y)} d \tau\right)^{2} \leq \int_{0}^{1} \Phi((1-\tau) x+\tau y) d \tau \leq \phi(x, y),
$$

we conclude that $M \leq \sup _{\mathbb{R}^{N} \times \mathbb{R}^{N}}\left(\frac{1}{2} \varepsilon \phi(x, y)-\varepsilon \phi(x, y)\right) \leq 0$ in the case where $\bar{t}=0$.

To prove the non-positivity of $M$ in the other case, we assume the contrary and we seek a contradition. In particular, we have $u(\bar{t}, \bar{x}) \geq u(\bar{t}, \bar{y})$. Moreover, recall that Lemma 2.3.2 yields the existence of

$$
\begin{aligned}
& \left(a, p+\varepsilon e^{\gamma \bar{t}} \nabla_{x} \phi(\bar{x}, \bar{y})\right) \in \bar{\partial}^{P} u(\bar{t}, \bar{x}), \\
& \left(b, p-\varepsilon e^{\gamma \bar{t}} \nabla_{y} \phi(\bar{x}, \bar{y})\right) \in \bar{\partial}_{P} v(\bar{t}, \bar{y})
\end{aligned}
$$

with $a-b=\gamma e^{\gamma \bar{t}} \frac{|\bar{x}-\bar{y}|^{2}}{2 \varepsilon}+\gamma \varepsilon e^{\gamma \bar{t}} \phi(\bar{x}, \bar{y}), p=e^{\gamma \bar{t}}\left(\frac{\bar{x}-\bar{y}}{\varepsilon}\right)$ and

$$
\begin{aligned}
& g[v]\left(\bar{t}, \bar{y}, p-\varepsilon e^{\gamma \bar{t}} \nabla_{y} \phi(\bar{x}, \bar{y})\right)-g[u]\left(\bar{t}, \bar{x}, p+\varepsilon e^{\gamma \bar{t}} \nabla_{x} \phi(\bar{x}, \bar{y})\right) \\
& \leq \varepsilon e^{\gamma \bar{t}} \int_{\mathbb{R}^{N}}\left(\phi(\bar{x}+z, \bar{y}+z)-\phi(\bar{x}, \bar{y})-\frac{\left(\nabla_{x}+\nabla_{y}\right) \phi(\bar{x}, \bar{y}) \cdot z}{1+|z|^{2}}\right) d \mu(z)=: \varepsilon e^{\gamma \bar{t}} J .
\end{aligned}
$$

By the definition of $\phi$, simple computations show that the integral term $J$ above equals

$$
J=J(\bar{x}, \bar{y})=\int_{0}^{1} g[\Phi]((1-\tau) \bar{x}+\tau \bar{y}) d \tau+g[\Phi](\bar{x})+g[\Phi](\bar{y}) .
$$

Using property (2.10) of barrier functions, we see that $|g[\Phi]| \leq C_{N, \lambda} \Phi$ and we obtain

$$
J(\bar{x}, \bar{y}) \leq \int_{0}^{1} C_{N, \lambda} \Phi((1-\tau) \bar{x}+\tau \bar{y}) d \tau+C_{N, \lambda} \Phi(\bar{x})+C_{N, \lambda} \Phi(\bar{y})=C_{N, \lambda} \phi(\bar{x}, \bar{y}) .
$$


It follows that the non-local terms satisfy

$$
g[v]\left(\bar{t}, \bar{y}, p-\varepsilon e^{\gamma \bar{t}} \nabla_{y} \phi(\bar{x}, \bar{y})\right)-g[u]\left(\bar{t}, \bar{x}, p+\varepsilon e^{\gamma \bar{t}} \nabla_{x} \phi(\bar{x}, \bar{y})\right) \leq C_{N, \lambda} \varepsilon e^{\gamma \bar{t}} \phi(\bar{x}, \bar{y}) .
$$

The viscosity inequalities and $\left(\mathrm{A} 1^{\prime}\right)$ now give

$$
\begin{aligned}
\gamma e^{\gamma \bar{t}} \frac{|\bar{x}-\bar{y}|^{2}}{2 \varepsilon}+\gamma \varepsilon e^{\gamma \bar{t}} \phi(\bar{x}, \bar{y}) & \leq H\left(\bar{t}, \bar{y}, u(\bar{t}, \bar{x}), p-\varepsilon e^{\gamma \bar{t}} \nabla_{y} \phi(\bar{x}, \bar{y})\right) \\
& -H\left(\bar{t}, \bar{x}, u(\bar{t}, \bar{x}), p+\varepsilon e^{\gamma \bar{t}} \nabla_{x} \phi(\bar{x}, \bar{y})\right)+C_{N, \lambda} \varepsilon e^{\gamma \bar{t}} \phi(\bar{x}, \bar{y}) .
\end{aligned}
$$

Using $\left(\mathrm{C} 3^{\prime}\right)$, we get

$$
\begin{aligned}
\gamma e^{\gamma \bar{t}} \frac{|\bar{x}-\bar{y}|^{2}}{2 \varepsilon}+\gamma \varepsilon e^{\gamma \bar{t}} \phi(\bar{x}, \bar{y}) \leq & H(\bar{t}, \bar{y}, u(\bar{t}, \bar{x}), p)-H(\bar{t}, \bar{x}, u(\bar{t}, \bar{x}), p) \\
& +C_{2} \varepsilon e^{\gamma \bar{t}}\left|\left(\nabla_{x}+\nabla_{y}\right) \phi(\bar{x}, \bar{y})\right|+C_{N, \lambda} \varepsilon e^{\gamma \bar{t}} \phi(\bar{x}, \bar{y}) .
\end{aligned}
$$

Using the fact that $\left|\left(\nabla_{x}+\nabla_{y}\right) \phi(\bar{x}, \bar{y})\right| \leq C_{N, \lambda} \phi(\bar{x}, \bar{y})$, thanks again to property (2.10) of barrier functions, we deduce that

$$
\begin{aligned}
\gamma e^{\gamma \bar{t}} \frac{|\bar{x}-\bar{y}|^{2}}{2 \varepsilon}+\gamma \varepsilon e^{\gamma \bar{t}} \phi(\bar{x}, \bar{y}) \leq & H(\bar{t}, \bar{y}, u(\bar{t}, \bar{x}), p)-H(\bar{t}, \bar{x}, u(\bar{t}, \bar{x}), p) \\
& +\left(1+C_{2}\right) C_{N, \lambda} \varepsilon e^{\gamma \bar{t}} \phi(\bar{x}, \bar{y}) .
\end{aligned}
$$

Since $\Phi \geq \Theta^{2}$, assumption (C2) implies that

$$
\gamma e^{\gamma \bar{t}} \frac{|\bar{x}-\bar{y}|^{2}}{2 \varepsilon}+\gamma \varepsilon e^{\gamma \bar{t}} \phi(\bar{x}, \bar{y}) \leq\left(I(\bar{x}, \bar{y})+C_{1}|p|\right)|\bar{x}-\bar{y}|+\left(1+C_{2}\right) C_{N, \lambda} \varepsilon e^{\gamma \bar{t}} \phi(\bar{x}, \bar{y}),
$$

where we recall that $I(x, y):=\int_{0}^{1} \sqrt{\Phi((1-\tau) x+\tau y)} d \tau$. Using the fact that $I \leq \sqrt{\phi}$, we get

$$
\begin{aligned}
\gamma e^{\gamma \bar{t}} \frac{|\bar{x}-\bar{y}|^{2}}{2 \varepsilon}+\gamma \varepsilon e^{\gamma \bar{t}} \phi(\bar{x}, \bar{y}) \leq & \sqrt{\phi(\bar{x}, \bar{y})}|\bar{x}-\bar{y}|+C_{1} e^{\gamma \bar{t}} \frac{|\bar{x}-\bar{y}|^{2}}{\varepsilon} \\
& +\left(1+C_{2}\right) C_{N, \lambda} \varepsilon e^{\gamma \bar{t}} \phi(\bar{x}, \bar{y}) .
\end{aligned}
$$

Rearranging terms, we get

$$
\left(\gamma-\left(1+C_{2}\right) C_{N, \lambda}\right) \varepsilon e^{\gamma \bar{t}} \phi(\bar{x}, \bar{y}) \leq \sqrt{\phi(\bar{x}, \bar{y})}|\bar{x}-\bar{y}|+\left(C_{1}-\gamma / 2\right) e^{\gamma \bar{t}} \frac{|\bar{x}-\bar{y}|^{2}}{\varepsilon} .
$$

Now take $\gamma:=\max \left\{2\left(C_{1}+1\right),\left(1+C_{2}\right) C_{N, \lambda}+1\right\}$. We get

$$
\varepsilon \phi(\bar{x}, \bar{y}) \leq \sqrt{\phi(\bar{x}, \bar{y})}|\bar{x}-\bar{y}|-\frac{|\bar{x}-\bar{y}|^{2}}{\varepsilon} \leq \frac{1}{2} \varepsilon \phi(\bar{x}, \bar{y}),
$$

which yields the desired contradiction since $\phi$ is positive. We then have proved that $M \leq 0$.

We end this section by studying how (1.1) keeps memory of the fact that the pure first order Hamilton-Jacobi equation propagates perturbations of the initial conditions with finite speed. In order to simplify the proof of the result below, we shall assume in addition that

(C1) $H$ does not depend on $u$. 
We then have the following result.

Theorem 3.10 (Finite-infinite propagation speed). Assume (C1),(B2),(C3'). Let $u$ and $v$ be, respectively, a sub- and supersolution of (1.1) such that $u$ and $v$ are $\mu_{b}$-uniformly integrable in space uniformly wrt time. Assume also that $u(0, \cdot)$ and $v(0, \cdot)$ are continuous. Then for all $t \in(0, T]$ and all $x \in \mathbb{R}^{N}$,

$$
u(t, x)-v(t, x) \leq K(t, \cdot) * \sup _{B_{C_{2} t^{(\cdot)}}}(u(0, \cdot)-v(0, \cdot))(x),
$$

where $C_{2}$ is the constant in $\left(\mathrm{C} 3^{\prime}\right)$.

Proof. Notice first that the right-hand side of (3.6) is well-defined, thanks to Lemma 2.5.1 and to the uniform integrability of $u(0, \cdot)$ and $v(0, \cdot)$ wrt $\mu_{b}$. Now, we let the reader check that $w=u-v$ is a viscosity subsolution of the equation

$$
\partial_{t} w+g[w]=C_{2}|\nabla w| \text {. }
$$

By the Lax-Oleinik formula, we know that $\alpha(t, x):=\sup _{B_{C_{2} t}(x)}(u(0, \cdot)-v(0, \cdot))$ is the unique continuous viscosity solution of

$$
\partial_{t} w=C_{2}|\nabla w|
$$

such that $w(0, \cdot)=u(0, \cdot)-v(0, \cdot)$. Consider a sequence $\left(w_{n}^{0}\right)_{n}$ of $W^{1, \infty}$ initial conditions that converges locally uniformly to $w(0, \cdot)$. The reader can check that we can assume that $\left|w_{n}^{0}\right| \leq \Phi$ where $\Phi$ is $\mu_{b}$-uniformly integrable. Define $\alpha_{n}(t, x):=$ $\sup _{B_{C_{2} t}(x)} w_{n}^{0}$. By stability, $\alpha_{n} \rightarrow \alpha$ locally uniformly as $n \rightarrow+\infty$. Moreover, we know that $\alpha_{n}$ is Lipschitz continuous and thus satisfies (3.8) almost everywhere. Let $\left(\theta_{m}\right)_{m}$ and $\left(\rho_{m}\right)_{m}$ be mollifiers in time and space respectively. Assume that $\operatorname{supp}\left(\theta_{m}\right) \subset(-\infty, 0)$. Define

$$
\alpha_{n, m}(t, x)=\alpha_{n} *_{t, x}\left(\rho_{m} \theta_{m}\right)(t, x):=\int_{0}^{+\infty} \alpha_{n}(s, .) * \rho_{m}(x) \theta(t-s) d s .
$$

The function $\alpha_{n, m}$ is smooth and

$$
\begin{aligned}
\partial_{t} \alpha_{n, m} & =\partial_{t}\left(\alpha_{n} *_{t, x}\left(\rho_{m} \theta_{m}\right)\right)=\left(\partial_{t} \alpha_{n}\right) *_{t, x}\left(\rho_{m} \theta_{m}\right) \\
& =C_{2}\left|\nabla \alpha_{n}\right| *_{t, x}\left(\rho_{m} \theta_{m}\right) \geq C_{2}\left|\nabla \alpha_{n, m}\right| .
\end{aligned}
$$

This means that $\alpha_{n, m}$ is a supersolution of (3.8). Now using the fact that $\partial_{t} K+$ $g[K]=0$, we obtain

$$
\begin{aligned}
\partial_{t}\left(K(t, \cdot) * \alpha_{n, m}(t, .)\right)(x) & =-g\left[K(t, \cdot) * \alpha_{n, m}(t, .)\right](x)+K(t, \cdot) *\left(\partial_{t} \alpha_{n, m}(t, .)\right)(x) \\
\geq & -g\left[K(t, \cdot) * \alpha_{n, m}(t, .)\right](x)+C_{2} K(t, \cdot) *\left|\nabla \alpha_{n, m}(t, .)\right|(x) \\
\geq & -g\left[K(t, \cdot) * \alpha_{n, m}(t, .)\right](x)+C_{2}\left|\nabla\left(K(t, \cdot) * \alpha_{n, m}(t, .)\right)(x)\right| .
\end{aligned}
$$

Hence $K(t, \cdot) * \alpha_{n, m}(t,).(x)$ is a smooth supersolution of (3.7); moreover, it converges locally uniformly to $K(t, \cdot) * \alpha_{n}(t,).(x)$ as $m \rightarrow+\infty$. By stability, $K(t, \cdot) *$ $\alpha_{n}(t,).(x)$ is a supersolution of (3.7). Next write

$$
K(t, .) * \alpha_{n}(t, .)=\int_{\mathbb{R}^{N}} K(1, y) \alpha_{n}\left(t, x-t^{1 / \lambda} y\right) d y
$$

and conclude that the (local uniform) limit of this function as $t$ goes to $0^{+}$exists and equals $\alpha_{n}(0,)=.w_{n}^{0}$. Now taking the limit as $n \rightarrow+\infty$, using the barrier function $\Phi$, the estimate $\left|w_{n}^{0}\right| \leq \Phi$ and the dominated convergence theorem we see that $K(t, \cdot) * \alpha(t,).(x)$ is a supersolution of (3.7) that satisfies $\alpha(0,)=.w(0, \cdot)$. The 
comparison principle then yields $w(t, x) \leq K(t, \cdot) * \alpha(t,).(x)$; that is to say, (3.6) holds true.

\section{Regularizing EFFECT}

In this section, we focus on the range of exponents $\lambda \in(1,2)$ and we prove that the unbounded continuous (viscosity) solution of (1.1) we constructed in the previous section is in fact $C^{1,1}$ with respect to $x$ and Lipschitz continuous with respect to $t$.

4.1. The sublinear case. In order to get the $C^{1,1}$ solution for (1.1), we need to strengthen assumptions. In particular, we need the following one:

$\left(\mathrm{A} 3^{\prime}\right)$ For all $R>0$, there exists a constant $C_{R}$ such that for all $x \in \mathbb{R}^{N}, u, v \in \mathbb{R}$, $p, q \in B_{R}$, and $t \in[0, T]$,

$$
|H(t, x, u, p)-H(t, x, v, q)| \leq C_{R}(|u-v|+|p-q|) .
$$

Theorem 4.1. Let $\lambda \in(1,2)$. Assume (A1), (A2'), and (A3') and consider a Lipschitz continuous function $u_{0}$. Then the (unique) viscosity solution of (1.1)(1.2) is $C^{1,1}$ wrt the space variable and locally Lipschitz continuous wrt the time variable on $(0, T] \times \mathbb{R}^{N}$.

Proof. Let us regularize the non-linearity and the initial data in order to ensure, thanks to the results of [25], existence and uniqueness of $C^{2}$ in space and $C^{1}$ in time bounded solution of (1.1). Let us consider $u_{n}^{0} \in W^{1, \infty}\left(\mathbb{R}^{N}\right)$ which converges locally uniformly to $u_{0}$ on $\mathbb{R}^{N}$ by satisfying

$$
\left\|u_{n}^{0}\right\|_{0,1}+\left\|\nabla u_{n}^{0}\right\|_{\infty} \leq C
$$

for some constant $C$. Consider a sequence of smooth non-linearities $H_{n}$ which converges locally uniformly to $H$ in $[0, T] \times \mathbb{R}^{N} \times \mathbb{R} \times \mathbb{R}^{N}$ by satisfying the assumptions of Theorem 4.1 (with some constants independent of $n$ ). Let $u_{n}$ be the viscosity solution associated with $H_{n}$ and $u_{n}^{0}$. The estimates of Theorems 3.2 and 3.3 imply that there exists a constant $M>0$ such that

$$
\left\|u_{n}\right\|_{0,1}+\left\|\nabla u_{n}\right\|_{\infty} \leq M
$$

This gives us in particular an $L_{l o c}^{\infty}$ estimate; hence, we can argue as in the proof of Theorem 3.8 to establish the local uniform convergence on $\bar{Q}_{T}$ of $u_{n}$ toward the unique $C_{\lambda^{\prime}}^{0}$ viscosity solution $u$ of (1.1)-(1.2). It thus remains to derive a $C^{1,1}$ in the space estimate on $u_{n}$.

In order to use the results of [25], we need to consider $H_{n}$ that satisfies in addition:

$$
\begin{aligned}
\sup _{\bar{Q}_{T}}\left|H_{n}(t, x, 0,0)\right| & <+\infty, \\
\forall R>0, \quad \sup _{[0, T] \times \mathbb{R}^{N} \times[-R, R] \times B_{R}}\left(\left|\partial_{u} H_{n}\right|\right. & +\left|\nabla_{p} H_{n}\right|+\left|D_{p, x}^{2} H_{n}\right| \\
& \left.+\left|\nabla_{p} \partial_{u} H_{n}\right|+\left|D_{p}^{2} H_{n}\right|\right)<+\infty .
\end{aligned}
$$

Then, $u_{n} \in C_{b}\left(\bar{Q}_{T}\right) \cap L^{\infty}\left([0, T] ; W^{1, \infty}\left(\mathbb{R}^{N}\right)\right)$ is $C^{2}$ in space and $C^{1}$ in time on $(0, T] \times \mathbb{R}^{N}$ with

$$
\sup _{[0, T]}\left\|t^{1 / \lambda} D^{2} u_{n}(t, .)\right\|_{\infty}<+\infty
$$


Moreover, $u_{n}$ satisfies Duhamel's Formula: for all $t>0$ and all $x \in \mathbb{R}^{N}$,

$$
u_{n}(t, x)=K(t, \cdot) * u_{n}^{0}(x)-\int_{0}^{t} K(t-s, .) * H_{n}\left(s, ., u_{n}(s, .), \nabla u_{n}(s, .)\right)(x) d s .
$$

By (2.6) and the properties of $u_{n}$ and $H_{n}$, the theorem of derivation under the integral sign implies that

$$
\begin{aligned}
D^{2} u_{n}(t, x)=\nabla & K(t, \cdot) * \nabla u_{n}^{0}(x)-\int_{0}^{t} \nabla K(t-s, .) \\
& * \nabla\left(H_{n}\left(s, ., u_{n}(s, .), \nabla u_{n}(s, .)\right)\right)(x) d s .
\end{aligned}
$$

Next compute

$$
\begin{aligned}
\nabla\left(H_{n}\left(s, ., u_{n}(s, .), \nabla u_{n}(s, .)\right)\right)= & \nabla_{x} H_{n}\left(s, . . u_{n}(s, .), \nabla u_{n}(s, .)\right) \\
& +\partial_{u} H_{n}\left(s, . . u_{n}(s, .), \nabla u_{n}(s, .)\right) \nabla u_{n}(s, .) \\
& +D^{2} u_{n}(s, .) \nabla_{p} H_{n}\left(s, ., u_{n}(s, .), \nabla u_{n}(s, .)\right) .
\end{aligned}
$$

By (4.1), $\left(\mathrm{A} 2^{\prime}\right)$ and $\left(\mathrm{A} 3^{\prime}\right)$, this implies

$$
\left\|\nabla\left(H_{n}\left(s, ., u_{n}(s, .), \nabla u_{n}(s, .)\right)\right)(x)\right\|_{\infty} \leq C_{1}(1+M)+C_{M}\left(M+\left\|D^{2} u_{n}(s, .)\right\|_{\infty}\right) .
$$

By (2.6), we get

$$
\begin{aligned}
& \left\|\nabla K(t-s, .) * \nabla\left(H_{n}\left(s, ., u_{n}(s, .), \nabla u_{n}(s, .)\right)\right)\right\|_{\infty} \\
\leq & (t-s)^{-1 / \lambda} \mathcal{K}_{0}\left[C_{1}(1+M)+C_{M} M\right]+(t-s)^{-1 / \lambda} s^{-1 / \lambda} \mathcal{K}_{0} C_{M}\left\|s^{1 / \lambda} D^{2} u_{n}(s, .)\right\|_{\infty} .
\end{aligned}
$$

Since $\left\|\nabla K(t, \cdot) * \nabla u_{n}^{0}\right\|_{\infty} \leq t^{-1 / \lambda} \mathcal{K}_{0} M$, we deduce from (4.4) that

$$
\begin{aligned}
\left\|t^{1 / \lambda} D^{2} u_{n}(t, .)\right\|_{\infty} \leq & \mathcal{K}_{0} M+t^{1 / \lambda} \int_{0}^{t}(t-s)^{-1 / \lambda} d s \mathcal{K}_{0}\left[C_{1}(1+M)+C_{M} M\right] \\
& +t^{1 / \lambda} \int_{0}^{t}(t-s)^{-1 / \lambda} s^{-1 / \lambda} d s \mathcal{K}_{0} C_{M} \sup _{\tau \in[0, T]}\left\|\tau^{1 / \lambda} D^{2} u_{n}(\tau, .)\right\|_{\infty} \\
= & \mathcal{K}_{0} M+\frac{\lambda}{1-\lambda} t \mathcal{K}_{0}\left[C_{1}(1+M)+C_{M} M\right] \\
& +I_{\lambda} t^{\frac{\lambda-1}{\lambda}} \mathcal{K}_{0} C_{M} \sup _{\tau \in[0, T]}\left\|\tau^{1 / \lambda} D^{2} u_{n}(\tau, .)\right\|_{\infty},
\end{aligned}
$$

where $I_{\lambda}=\int_{0}^{1}(1-s)^{-1 / \lambda} s^{-1 / \lambda} d s$. Let $T_{1} \in(0, T]$ be such that $I_{\lambda} T_{1}^{\frac{\lambda-1}{\lambda}} \mathcal{K}_{0} C_{M} \leq$ $1 / 2$. We have proved that

$$
\begin{aligned}
\sup _{\left[0, T_{1}\right]}\left\|t^{1 / \lambda} D^{2} u_{n}(t, .)\right\|_{\infty} & \leq 2\left(\mathcal{K}_{0} M+\frac{\lambda}{1-\lambda} T_{1} \mathcal{K}_{0}\left[C_{1}(1+M)+C_{M} M\right]\right) \\
& =: C\left(M, \lambda, \mathcal{K}_{0}\right) .
\end{aligned}
$$

Taking any $T_{0}$ as initial time, we can argue similarly to prove that for all $n \in \mathbb{N}$ and all $T_{0} \in\left[0, T-T_{1}\right]$,

$$
\sup _{\left[T_{0}, T_{0}+T_{1}\right]}\left\|\left(t-T_{0}\right)^{1 / \lambda} D^{2} u_{n}(t, .)\right\|_{\infty} \leq C\left(M, \lambda, \mathcal{K}_{0}\right)
$$

Eventually, we get for all $t \in[0, T]$ and $x \in \mathbb{R}^{N}$,

$$
\left|D^{2} u_{n}(t, x)\right| \leq t^{-1 / \lambda} \tilde{C}\left(M, \lambda, \mathcal{K}_{0}\right) .
$$


This gives the desired $C^{1,1}$ estimate in space. The equation

$$
\partial_{t} u_{n}=-H_{n}\left(t, x, u_{n}, \nabla u_{n}\right)-g\left[u_{n}\right]
$$

now allows us to derive a Lipschitz continuous in time estimate on $u_{n}$. The proof is complete.

Corollary 4.1 (Duhamel's Formula). The unique viscosity solution of (1.1)-(1.2) satisfies

$$
u(t, x)=K(t, \cdot) * u_{0}(x)-\int_{0}^{t} K(t-s, .) * H(s, ., u(s, .), \nabla u(s, .))(x) d s .
$$

Proof. We need to check that we can pass to the limit in (4.3). This reduces to obtain pointwise convergence of gradients. To prove this, remark that we have the $C^{1,1}$ estimate (4.5) and $\left\|\nabla u_{n}\right\|_{\infty} \leq M$. Hence, we have compactness. The fact that $u_{n}$ converges locally uniformly towards $u$ tells us that the limit of any subsequence of $\nabla u_{n}$ has to be $\nabla u$. Indeed, it coincides with the distribution limit.

4.2. The superlinear case. To get the $C^{1}$ and/or $C^{1,1}$ regularity in the superlinear case, we need to strengthen assumption (A1).

(B1) There exists a constant $C_{3}$ such that for all $x \in \mathbb{R}^{N}, u \in \mathbb{R}, p \in \mathbb{R}^{N}$, and $t \in[0, T]$

$$
|H(t, x, u, p)-H(t, x, v, p)| \leq C_{3}|u-v| .
$$

It turns out that the assumptions that permit us to get gradient estimate for a viscosity solution in the superlinear case (see the assumptions of Theorem 3.6) only permit us to prove $C^{1}$ regularity in space; moreover, initial conditions have to be locally Lipschitz continuous; precisely, they have to lie in $C_{\lambda^{\prime}}^{0,1}$. If now one considers the assumptions that permit us to get a gradient estimate in the case of finite-infinite speed of propagation (Theorem [3.9), solutions can be proved to be $C^{1,1}$. Moreover, initial conditions do not need to be assumed to be locally Lipschitz continuous; precisely, they have to lie in $C_{\lambda^{\prime}}^{0}$. Let us first state both results before turning to their proofs.

Theorem 4.2. Let $\lambda \in(1,2)$ and $\lambda^{\prime} \in(1, \lambda)$. Assume (B1), (B2'), (B3'), and (A4). Let $u_{0} \in C_{\lambda^{\prime}}^{0,1}\left(\mathbb{R}^{N}\right)$. Then the (unique) viscosity solution of (1.1)-(1.2) is $C^{1}$ wrt the space variable on $(0, T] \times \mathbb{R}^{N}$.

Remark 4.1. Notice that $\left(\mathrm{B} 2^{\prime}\right)$ and the continuity of $H(t, 0,0,0)$ wrt $t \in[0, T]$ already imply (A4).

Remark 4.2. Duhamel's Formula holds true in this case too (see Corollary 4.1) under the assumptions of Theorem 4.2 .

Theorem 4.3. Let $\lambda \in(1,2)$ and $\lambda^{\prime} \in(1, \lambda)$. Assume (B1), (B2'), $\left(\mathrm{C} 3^{\prime}\right)$, and (A4). Let $u_{0} \in C_{\lambda^{\prime}}^{0}\left(\mathbb{R}^{N}\right)$. Then the (unique) viscosity solution of (1.1)-(1.2) is $C^{1,1}$ wrt the space variable and locally Lipschitz continuous wrt the time variable in $(0, T] \times \mathbb{R}^{N}$.

Proof of Theorem 4.2, Following the proof of Theorem 4.1, we first regularize the non-linearity and the initial data in such a way that $\left\|u_{n}^{0}\right\|_{1, \lambda^{\prime}}$ remains bounded. We have a $C_{\lambda^{\prime}}^{0,1}$ bound on $\left(u_{n}\right)_{n}$ given by Theorems 3.5 and 3.6. Let $M$ denote 
this bound. By the theorem of derivation under the integral sign and Duhamel's Formula (4.3),

$\nabla u_{n}(t, x)=\nabla K(t, \cdot) * u_{n}^{0}(x)-\int_{0}^{t} \nabla K(t-s,). * H_{n}\left(s, x, u_{n}(s,),. \nabla u_{n}(s,).\right)(x) d s$.

Define $\mathcal{H}_{n}(s, x):=H_{n}\left(s, x, u_{n}(s, x), \nabla u_{n}(s, x)\right) \mathbf{1}_{(0, T)}(s) \quad$ and $\quad \mathcal{K}(s, x) \quad:=$ $\nabla K(s, x) \mathbf{1}_{(0, T)}(s)$. The last term on the right-hand side of (4.6) is equal to $\mathcal{H}_{n} * \mathcal{K}$, where the convolution is computed wrt $(t, x)$. Assumptions (A4), (B1) and (B3') imply

$$
\left\|\mathcal{H}_{n}\right\|_{0, \lambda^{\prime}} \leq C_{0}+4 C_{3} M
$$

By (2.6), $\mathcal{K}$ is integrable with respect to the measure $\left(1+|z|^{\lambda^{\prime}}\right) d \tau d z$. Hence, the continuity of the translations in $L^{1}\left(\left(1+|z|^{\lambda^{\prime}}\right) d \tau d z\right)$ implies that $\left(\mathcal{H}_{n} * \mathcal{K}\right)_{n}$ is equicontinuous in $\bar{Q}_{T}$; indeed, we can write

$$
\begin{aligned}
\left|\mathcal{H}_{n} * \mathcal{K}(t, x)-\mathcal{H}_{n} * \mathcal{K}(s, y)\right| \leq & \left\|\mathcal{H}_{n}\right\|_{0, \lambda^{\prime}} \int_{\mathbb{R}} \int_{\mathbb{R}^{N}}|\mathcal{K}(\tau+(t-s), z+(x-y))-\mathcal{K}(\tau, z)| \\
& \times\left(1+|z|^{\lambda^{\prime}}\right) d \tau d z \\
\leq & \left(C_{0}+4 C_{3} M\right) \omega(|t-s|+|x-y|),
\end{aligned}
$$

where $\omega$ is a modulus of continuity. Now using the bound on $\left\|u_{n}^{0}\right\|_{0, \lambda^{\prime}}$, it is immediate to see that the sequence $\left(\nabla K(t, \cdot) * u_{n}^{0}\right)_{n}$ is equicontinuous in $(0, T] \times \mathbb{R}^{N}$. Hence, so is $\left(\nabla u_{n}\right)_{n}$ and the local uniform limit $u$ of $u_{n}$ is $C^{1}$ in space in $(0, T] \times \mathbb{R}^{N}$. The proof of Theorem 4.2 is complete.

Proof of Theorem 4.3. Once again, we regularize the initial conditions as in Theorem 4.1. In particular, the corresponding solution of (1.1) remains bounded in $C_{\lambda^{\prime}}^{0}$ and $M$ denotes a bound. Let us derive a local gradient estimate on $\nabla u_{n}$ with the help of (4.6). By (A4), (B1) and (C3),

$$
\left\|H_{n}\left(s, ., u_{n}(s, .), \nabla u_{n}(s, .)\right)\right\|_{0, \lambda^{\prime}} \leq C_{0}+C_{3} M+C_{2}\left\|\nabla u_{n}(s, .)\right\|_{0, \lambda^{\prime}} .
$$

By (2.6), we get

$$
\begin{aligned}
& \left\|\nabla K(t-s, .) * H_{n}\left(s, . . u_{n}(s, .), \nabla u_{n}(s, .)\right)\right\|_{0, \lambda^{\prime}} \\
& \quad \begin{array}{l}
\quad 2(t-s)^{-1 / \lambda}\left(\mathcal{K}_{0}+\mathcal{K}_{\lambda^{\prime}}\right)\left(C_{0}+C_{3} M\right) \\
\quad+2(t-s)^{-1 / \lambda} s^{-1 / \lambda}\left(\mathcal{K}_{0}+\mathcal{K}_{\lambda^{\prime}}\right) C_{2}\left\|s^{1 / \lambda} \nabla u_{n}(s, .)\right\|_{0, \lambda^{\prime}} .
\end{array}
\end{aligned}
$$

Since $\left\|\nabla K(t, \cdot) * u_{n}^{0}\right\|_{0, \lambda^{\prime}} \leq 2 t^{-1 / \lambda}\left(\mathcal{K}_{0}+\mathcal{K}_{\lambda^{\prime}}\right) M$, we deduce from (4.6) that

$$
\begin{aligned}
\left\|t^{1 / \lambda} \nabla u_{n}(t, .)\right\|_{0, \lambda^{\prime}} \leq 2\left(\mathcal{K}_{0}+\mathcal{K}_{\lambda^{\prime}}\right) M+2 \frac{\lambda}{1-\lambda} t\left(\mathcal{K}_{0}+\mathcal{K}_{\lambda^{\prime}}\right)\left(C_{0}+C_{3} M\right) \\
+2 I_{\lambda} t^{\frac{\lambda-1}{\lambda}}\left(\mathcal{K}_{0}+\mathcal{K}_{\lambda^{\prime}}\right) C_{2} \sup _{[0, T]}\left\|s^{1 / \lambda} \nabla u_{n}(s, .)\right\|_{0, \lambda^{\prime}} .
\end{aligned}
$$

Let $T_{1} \in[0, T]$ be such that $2 I_{\lambda} T_{1}^{\frac{\lambda-1}{\lambda}}\left(\mathcal{K}_{0}+\mathcal{K}_{\lambda^{\prime}}\right) C_{2} \leq 1 / 2$. We have proved that

$$
\sup _{\left[0, T_{1}\right]}\left\|t^{1 / \lambda} \nabla u_{n}(t, .)\right\|_{0, \lambda^{\prime}} \leq 4\left(\left(\mathcal{K}_{0}+\mathcal{K}_{\lambda^{\prime}}\right) M+\frac{\lambda}{1-\lambda} T_{1}\left(\mathcal{K}_{0}+\mathcal{K}_{\lambda^{\prime}}\right)\left(C_{0}+C_{3} M\right)\right) .
$$


Taking any $T_{0}$ as initial time, we can argue similarly to prove that for all $n \in \mathbb{N}$ and all $T_{0} \in\left[0, T-T_{1}\right]$

$$
\begin{aligned}
& \sup _{\left[T_{0}, T_{0}+T_{1}\right]}\left\|\left(t-T_{0}\right)^{1 / \lambda} \nabla u_{n}(t, .)\right\|_{0, \lambda^{\prime}} \\
& \quad \leq 4\left(\left(\mathcal{K}_{0}+\mathcal{K}_{\lambda^{\prime}}\right) M+\frac{\lambda}{1-\lambda} T_{1}\left(\mathcal{K}_{0}+\mathcal{K}_{\lambda^{\prime}}\right)\left(C_{0}+C_{3} M\right)\right) .
\end{aligned}
$$

The local uniform limit $u$ of $u_{n}$ is then locally Lipschitz continuous wrt $x$ in $(0, T] \times$ $\mathbb{R}^{N}$. This process can be iterated with the formula (4.4) to prove the $C^{1,1}$ regularity in space and a fortiori the local Lipschitz continuity regularity in time. The proof of Theorem 4.3 is now complete.

Let us end this section with a remark on regularity results in the uniformly integrable framework.

Remark 4.1. The key property on the kernel used in the preceding proof is estimate (2.6). By the homogeneity property of $K$, this property still holds true for $\left(1+|x|^{\lambda^{\prime}}\right)$ replaced by $\Phi(|x|)$, with $\Phi: \mathbb{R}^{+} \rightarrow \mathbb{R}^{+}$non-decreasing and such that $\int \Phi(|z|) d \mu_{b}(z)<+\infty$. This suggests that our techniques can be adapted to initial conditions satisfying (2.11).

\section{Convergence of GRadients as "Viscosity" VAnishes}

In this section, we prove a result about the sequence of the gradients of the solution $u^{\varepsilon}$ of (1.7)-(1.8) under the following assumption:

(D) The non-linearity $H$ is strictly convex wrt $p$.

Theorem 5.1. Let $\lambda \in(1,2)$ and $\lambda^{\prime} \in(1, \lambda)$. Let $u_{0} \in C_{\lambda^{\prime}}^{0,1}\left(\mathbb{R}^{N}\right)$ (resp. let $u_{0}$ be Lipschitz continuous). Assume (D) and the assumptions of Theorem 4.2 (resp. of Theorem 4.1). Then the unbounded regular solution $u^{\varepsilon}$ of (1.7)-(1.8) converges locally uniformly to the solution $u^{0}$ of (1.7) with $\varepsilon=0$ and the sequence of gradients satisfies: for all $p \in[1,+\infty)$,

$$
\nabla u^{\varepsilon}(t, x) \underset{\varepsilon \rightarrow 0}{\rightarrow} \nabla u^{0}(t, x) \quad \text { in } \quad L_{\mathrm{loc}}^{p}\left(\bar{Q}_{T}\right) .
$$

Proof. To avoid technicalities with Young measures, we only do the proof with $H(t, x, u, p)=H(p)$ and let the reader check that the following ideas can be adapted in the general case.

The kernel associated with $\varepsilon g[\cdot]$ is $K_{\varepsilon}(t, x)=K(\varepsilon t, x)$. We then have

$$
u^{\varepsilon}(t, x)=K(\varepsilon t, .) * u_{0}(x)+\int_{0}^{t} K(\varepsilon(t-s), \cdot) * H\left(\nabla u^{\varepsilon}(s, \cdot)\right) d s .
$$

Consider the Young measure $\nu$ associated with the locally bounded family $\left\{\nabla u^{\varepsilon}\right\}_{\varepsilon>0}$ with $\varepsilon \rightarrow 0$. Precisely, its disintegration is a family of probabilities $\left\{\nu_{t, x}\right\}_{(t, x) \in Q_{T}}$ such that for all $F \in C\left(\mathbb{R}^{N}\right)$ and all $\phi \in C_{c}\left(\bar{Q}_{T}\right)$,

$$
\begin{array}{r}
\int_{0}^{T} \int_{\mathbb{R}^{N}} F\left(\nabla u^{\varepsilon}(t, x)\right) \phi(t, x) d t d x \rightarrow \int_{0}^{T} \int_{\mathbb{R}^{N}} \int_{\mathbb{R}^{N}} F(\xi) \phi(t, x) d \nu_{t, x}(\xi) d t d x \\
\text { as } \quad \varepsilon \rightarrow 0
\end{array}
$$

(up to a subsequence). Indeed, Prokhorov's Theorem gives us the existence of a Young measure $\nu_{R}$ on $\mathbb{R}^{N}$ indexed by $(0, T) \times B_{R}$ for all $R>0$ associated 
with the bounded sequence $\left(\mathbf{1}_{(0, T) \times B_{R}} \nabla u^{\varepsilon}\right)_{\varepsilon>0}$. An argument of diagonalization (with $R_{n}=n$ for instance) then implies the existence of the measure $\nu$ indexed by $Q_{T}$ that coincides with $\nu_{R}$ on each $(0, T) \times B_{R}$. Notice finally, that by the local bound we have on $\nabla u^{\varepsilon}$ (see Theorems 3.3 and 3.6 respectively), (5.2) holds true for $F$ not necessarily bounded on $\mathbb{R}^{N}$ and $\varphi$ with compact support on $\bar{Q}_{T}$; indeed, $\operatorname{supp}\left(\nu_{t, x}\right) \subset\left\{|\xi| \leq \sup _{\varepsilon>0}\left|\nabla u^{\varepsilon}(t, x)\right|\right\}$. This implies that

$$
\int_{0}^{t} \int_{\mathbb{R}^{N}} F\left(\nabla u^{\varepsilon}(s, x)\right) d s \phi(x) d x \rightarrow \int_{0}^{t} \int_{\mathbb{R}^{N}} \int_{\mathbb{R}^{N}} F(\xi) d \nu_{s, x}(\xi) d s \phi(x) d x \quad \text { as } \varepsilon \rightarrow 0 .
$$

We will use it with $F=H$. The previous convergence result implies that

$$
\int_{0}^{t} H\left(\nabla u^{\varepsilon}(s, x)\right) d s \rightarrow \int_{0}^{t} \int_{\mathbb{R}^{N}} H(\xi) d \nu_{s, x}(\xi) d s \quad \text { in } \quad \mathcal{D}^{\prime}\left(\mathbb{R}^{N}\right) .
$$

We next prove that for almost every (a.e. for short) $(t, x) \in Q_{T}, \nu_{t, x}$ is a Dirac mass centered at $\nabla u^{0}(t, x)$. Classical results about Young measures will thus imply the convergence in $L_{\mathrm{loc}}^{p}\left(\bar{Q}_{T}\right)$ for all $p \geq 1$.

We claim that, as $\varepsilon \rightarrow 0$,

$$
\int_{0}^{t} K(\varepsilon(t-s), \cdot) * H\left(\nabla u^{\varepsilon}(s, \cdot)\right)(x) d s \rightarrow \int_{0}^{t} \int_{\mathbb{R}^{N}} H(\xi) d \nu_{s, x}(\xi) d s \quad \text { in } \quad \mathcal{D}^{\prime}\left(\mathbb{R}^{N}\right) .
$$

Indeed, thanks to the evenness of $K$ wrt $x$, we have

$$
\begin{aligned}
& \int_{0}^{t} \int_{\mathbb{R}^{N}} K(\varepsilon(t-s), \cdot) * H(\left.\nabla u^{\varepsilon}(s, \cdot)\right)(x) \varphi(x) d x d s \\
&=\int_{0}^{t} \int_{\mathbb{R}^{N}} K(\varepsilon(t-s), \cdot) * \varphi(x) H\left(\nabla u^{\varepsilon}(s, x)\right) d x d s .
\end{aligned}
$$

Recalling that $\int_{\mathbb{R}^{N}} K(t, \cdot)=1$, the homogeneity property of $K$ implies from the equality above that

$$
\begin{aligned}
& \left|\int_{0}^{t} \int_{\mathbb{R}^{N}} K(\varepsilon(t-s), \cdot) * H\left(\nabla u^{\varepsilon}(s, .)\right)(x) \varphi(x) d x d s-\int_{0}^{t} \int_{\mathbb{R}^{N}} H\left(\nabla u^{\varepsilon}(s, x)\right) \varphi(x) d x d s\right| \\
& \quad \leq \int_{0}^{t} \int_{\mathbb{R}^{N}} \int_{\mathbb{R}^{N}} K(\varepsilon(t-s), z)|\varphi(x-z)-\varphi(x)|\left|H\left(\nabla u^{\varepsilon}(s, x)\right)\right| d z d x d s \\
& \quad \leq \int_{0}^{t} \int_{\mathbb{R}^{N}} \int_{\mathbb{R}^{N}} K(t-s, z)\left|\varphi\left(x-\varepsilon^{1 / \lambda} z\right)-\varphi(x)\right|\left|H\left(\nabla u^{\varepsilon}(s, x)\right)\right| d z d x d s \\
& \quad \leq \varepsilon^{1 / \lambda}\|\nabla \varphi\|_{L^{1}\left(\mathbb{R}^{N}\right)} \int_{0}^{t} \int_{\mathbb{R}^{N}} K(t-s, z)|z| \sup _{1 \geq \varepsilon>0} \sup _{|x| \leq R_{\varphi}+\varepsilon^{1 / \lambda}|z|}\left|H\left(\nabla u^{\varepsilon}(s, x)\right)\right| d z d s
\end{aligned}
$$

where $\operatorname{supp}(\varphi) \subset B_{R_{\varphi}}$. By (2.5), the growth conditions on $H$ and the local bound we have on $\nabla u^{\varepsilon}$, we deduce that the left hand side of (5.4) goes to 0 as $\varepsilon \rightarrow 0$.

Since $u^{\varepsilon}$ converges towards $u^{0}$ locally uniformly, we can pass to the limit in Duhamel's Formula (see Corollary 4.1] and Remark 4.2) in the distribution sense as $\varepsilon \rightarrow 0$. We thus obtain by using (5.3) that

$$
u^{0}(t, x)=u_{0}(x)+\int_{0}^{t} \int_{\mathbb{R}^{N}} H(\xi) d \nu_{s, x}(\xi) \quad \text { a.e. } \quad x .
$$


Since $u^{0}$ is locally Lipschitz continuous wrt $(t, x)$, we also have an integral (mild) formulation

$$
u^{0}(t, x)=u_{0}(x)+\int_{0}^{t} H\left(\nabla u^{0}(s, x)\right) d s \quad \text { a.e. } \quad x .
$$

Combining (5.5) and (5.6), we obtain

$$
\int_{0}^{t} \int_{\mathbb{R}^{N}} H(\xi) d \nu_{s, x}(\xi) d s=\int_{0}^{t} H\left(\nabla u^{0}(s, x)\right) d s \quad \text { a.e. } \quad x .
$$

Both sides are Lipschitz continuous wrt $t$, and we obtain for a.e. $(t, x)$

$$
\int_{\mathbb{R}^{N}} H(\xi) d \nu_{t, x}(\xi)=H\left(\nabla u^{0}(t, x)\right) .
$$

Now use the facts that $\int_{\mathbb{R}^{N}} \xi d \nu_{t, x}(\xi)=\nabla u^{0}(t, x)$ and that $H$ is strictly convex to conclude that $\nu_{t, x}=\delta_{\nabla u^{0}(t, x)}$. The proof is now complete.

\section{Appendix A. Sketches of proofs of Classical Results}

\section{A.1. Proofs of Lemmata 2.3.1 and 2.3.2.}

Proof of Lemma 2.3.1. Let $(a, p) \in \bar{\partial}^{P} u(t, x)$. Following [7, it is quite classical to pass to the limit in the Hamiltonian; hence, we only need to check that we can pass to the limit in the integral term. To do so, let $f_{n}(z)$ denote the function $u\left(t_{n}, x_{n}+z\right)-u\left(t_{n}, x_{n}\right)-\frac{p_{n} \cdot z}{1+|z|^{2}}$, where $t_{n}, x_{n}$ and $p_{n}$ appear in the definition of the closure of a superdifferential. Let $\Phi$ be the barrier function associated to $u \in \mathcal{C}^{+}$. By the definition of a supergradient, we have

$$
f_{n}(z) \leq \Phi\left(x_{n}+z\right) \mathbf{1}_{B_{r / 2}^{c}}(z)+g(z)
$$

for $n$ large enough, where $g$ is continuous and $\mu$-integrable. For $R>2|x|$, define

$$
\omega(R):=\int_{B_{R}^{c}} \Phi\left(x_{n}+z\right) d \mu(z)+\int_{B_{R}^{c}} g(z) d \mu(z),
$$

where

$$
\begin{aligned}
\int_{B_{R}^{c}} \Phi\left(x_{n}+z\right) d \mu(z) & =c_{N}(\lambda) \int_{B_{R}^{c}} \Phi\left(z^{\prime}\right) \frac{d z^{\prime}}{\left|z^{\prime}-x_{n}\right|^{1+\lambda}} \\
& \leq 2^{N+\lambda} c_{N}(\lambda) \int_{B_{R}^{c}} \Phi\left(z^{\prime}\right) \frac{d z^{\prime}}{\left|z^{\prime}\right|^{1+\lambda}}
\end{aligned}
$$

since $\left|z^{\prime}-x_{n}\right| \geq\left|z^{\prime}\right|-\left|x_{n}\right| \geq \frac{1}{2}\left|z^{\prime}\right|$ for $n$ large enough. By the integrability property of $\Phi$, we see that $\lim _{R \rightarrow+\infty} \omega(R)=0$. Moreover, Fatou's lemma implies

$$
\limsup _{n \rightarrow+\infty} \int_{\mathbb{R}^{N}} f_{n}(z) d \mu(z) \leq \int_{B_{R}} \limsup _{n \rightarrow+\infty} f_{n}(z) d \mu(z)+\omega(R) .
$$

The positive part of $\lim \sup _{n \rightarrow+\infty} f_{n}(z)$ is $\mu$-integrable since it is bounded above by $\Phi(x+z) \mathbf{1}_{B_{r}^{c}}+g(z)$; hence, we can again use Fatou's lemma to pass to the limit as $R \rightarrow+\infty$ and deduce that

$$
\limsup _{n \rightarrow+\infty} \int_{\mathbb{R}^{N}} f_{n}(z) d \mu(z) \leq \int_{\mathbb{R}^{N}} \limsup _{n \rightarrow+\infty} f_{n}(z) d \mu(z) .
$$


The limit equation then becomes

$$
a+H(t, x, u(t, x), p) \leq \int_{\mathbb{R}^{N}} \limsup _{n \rightarrow+\infty} f_{n}(z) d \mu(z) .
$$

The right-hand side of A.1 is finite, which implies that $\lim _{\sup } \operatorname{su}_{n \rightarrow+\infty} f_{n}(z)$ is $\mu$ integrable. Finally, the upper-semicontinuity of $u$ and the fact that $u_{n}\left(t_{n}, x_{n}\right) \rightarrow$ $u(t, x)$ imply

$$
\limsup _{n \rightarrow+\infty} f_{n}(z) \leq u(t, x+z)-u(t, x)-\frac{p \cdot z}{1+|z|^{2}}=: f(z) ;
$$

hence, the negative part of $f(z)$ is $\mu$-integrable and (A.1) gives

$$
a+H(t, x, u(t, x), p) \leq \int_{\mathbb{R}^{N}} f(z) d \mu(z) .
$$

The proof of Lemma 2.3.1 is complete.

Proof of Lemma 2.3.2. To prove the existence of $a$ and $b$, we let the reader check that we can slightly modify $\phi$, without changing its first order partial derivatives at $(\bar{t}, \bar{x}, \bar{y})$, in such a way that $M$ achieves a strict maximum at $(\bar{t}, \bar{x}, \bar{y})$ and $\phi$ is positive and such that

$$
\limsup _{|(x, y)| \rightarrow+\infty} \sup _{t \in[0, T]} \frac{u(t, x)-v(t, y)}{\phi(t, x, y)} \leq 0 .
$$

Consider a parameter $\delta>0$ and define

$$
M_{\delta}:=\sup _{[0, T] \times[0, T] \times \mathbb{R}^{N} \times \mathbb{R}^{N}}\left(u(t, x)-v(s, y)-\frac{|s-t|^{2}}{2 \delta}-\phi(t, x, y)\right) .
$$

This supremum is achieved at some point $\left(t_{\delta}, s_{\delta}, x_{\delta}, y_{\delta}\right)$. By assumption (A.2), the set $\left\{\left(t_{\delta}, s_{\delta}, x_{\delta}, y_{\delta}\right): \delta>0\right\}$ is relatively compact and we know that $\left(t_{\delta}, s_{\delta}, x_{\delta}, y_{\delta}\right) \rightarrow$ $(\bar{t}, \bar{t}, \bar{x}, \bar{y})$ as $\delta \rightarrow 0$. Moreover,

$$
\begin{aligned}
\left(a_{\delta}, \nabla_{x} \phi\left(t_{\delta}, x_{\delta}, y_{\delta}\right)\right) & \in \partial^{P} u\left(t_{\delta}, x_{\delta}\right), \\
\left(b_{\delta},-\nabla_{y} \phi\left(t_{\delta}, x_{\delta}, y_{\delta}\right)\right) & \in \partial_{P} v\left(s_{\delta}, y_{\delta}\right),
\end{aligned}
$$

where $b_{\delta}=\frac{t_{\delta}-s_{\delta}}{\delta}$ and $a_{\delta}=b_{\delta}+\partial_{t} \phi\left(t_{\delta}, x_{\delta}, y_{\delta}\right)$. By writing the viscosity inequality for $u$, we get

$$
a_{\delta} \leq-H\left(t_{\delta}, u\left(t_{\delta}, x_{\delta}\right), \nabla_{x} \phi\left(t_{\delta}, x_{\delta}, y_{\delta}\right)\right)-g[u]\left(t_{\delta}, x_{\delta}, \nabla_{x} \phi\left(t_{\delta}, x_{\delta}, y_{\delta}\right)\right) .
$$

One can see that the constants in the definition of the supergradient $\left(a_{\delta}, \nabla_{x} \phi\left(t_{\delta}, x_{\delta}, y_{\delta}\right)\right)$ can be chosen as follows:

$$
\sigma_{\delta}:=\sup _{\left|t-t_{\delta}\right|+\left|x-x_{\delta}\right|+\left|y-y_{\delta}\right| \leq 1}\left|D_{x}^{2} \phi(t, x, y)\right|
$$

and $r=1$. According to the growth condition on $u$, this gives us an upper bound on $-g[u]\left(t_{\delta}, x_{\delta}, \nabla_{x} \phi\left(t_{\delta}, x_{\delta}, y_{\delta}\right)\right)$ which does not depend on small $\delta$. The viscosity inequality above then gives us an upper bound on $a_{\delta}$ independently on small $\delta$. Similar arguments allow us to prove that $b_{\delta}$ is bounded from below independently on small $\delta$. Since $a_{\delta}-b_{\delta}=\partial_{t} \phi\left(t_{\delta}, x_{\delta}, y_{\delta}\right)$, we deduce that $a_{\delta}$ and $b_{\delta}$ are bounded 
independently on small $\delta$. There then exist $a, b \in \mathbb{R}$ such that $\left(a_{\delta}, b_{\delta}\right) \rightarrow(a, b)$ as $\delta \rightarrow 0$ (up to a subsequence). It is now straightforward that

$$
\begin{gathered}
\left(a, \nabla_{x} \phi(\bar{t}, \bar{x}, \bar{y})\right) \in \bar{\partial}^{P} u(\bar{t}, \bar{x}), \\
\left(b,-\nabla_{y} \phi(\bar{t}, \bar{x}, \bar{y})\right) \in \bar{\partial}_{P} v(\bar{t}, \bar{y})
\end{gathered}
$$

and $a-b=\partial_{t} \phi(\bar{t}, \bar{x}, \bar{y})$.

It remains to prove that (2.3) holds true. Writing that $M=u(\bar{t}, \bar{x})-v(\bar{t}, \bar{y})-$ $\phi(\bar{t}, \bar{x}, \bar{y})$, we get

$$
\begin{aligned}
u(\bar{t}, \bar{x}+z)-u(\bar{t}, \bar{x})- & \frac{\nabla_{x} \phi(\bar{t}, \bar{x}, \bar{y}) \cdot z}{1+|z|^{2}}-v(\bar{t}, \bar{y}+z)+v(\bar{t}, \bar{y})+\frac{\nabla_{y} \phi(\bar{t}, \bar{x}, \bar{y}) \cdot z}{1+|z|^{2}} \\
& \leq \phi(\bar{t}, \bar{x}+z, \bar{y}+z)-\phi(\bar{t}, \bar{x}, \bar{y})-\frac{\left(\nabla_{x}+\nabla_{y}\right) \phi(\bar{t}, \bar{x}, \bar{y}) \cdot z}{1+|z|^{2}}
\end{aligned}
$$

for all $z \in \mathbb{R}^{N}$. We have seen in the preceding proof that the negative part of the left-hand side of this inequality is $\mu$-integrable; hence, so is the negative part of the right-hand side, and integrating with respect to $d \mu(z)$ completes the proof of Lemma 2.3.2

\section{A.2. Main ideas of the proof of the discontinuous stability.}

Sketch of the proof of Proposition 2.1. Following the preceding proof of Lemma 2.3.1, one can easily adapt the ideas of [7] to prove that $u:=\limsup ^{*} u_{n}$ is a viscosity subsolution of (1.1) with generalized initial condition:

$$
\min \left\{\partial_{t} u(0, .)+H(0, x, u(0, .), \nabla u(0, .))+g[u(0, .)], u(0, .)-u_{0}\right\} \leq 0,
$$

where $u_{0}:=\lim \sup ^{*}\left(u_{n}(0,).\right)$; the main difficulty is to handle the non-local term, and this is done by using Fatou's lemma.

Let us prove (2.4). For $\lambda^{\prime} \in(0, \lambda), \varepsilon>0$ and $C>0$, define

$$
\chi(t, y)=u(t, y)-\frac{\phi_{\varepsilon}(y-x)}{\varepsilon}-\Phi(y)-C t,
$$

where $\phi_{\varepsilon}(z):=\frac{\left(\varepsilon+|z|^{2}\right)^{\frac{\lambda^{\prime}}{2}}}{\lambda^{\prime}}$. Using some ideas from the proof of Lemma 2.5.2, we claim that $\Phi$ can be slightly modified in such a way that $\Phi$ stays positive, is $C^{2}$ and $\mu_{b}$-integrable, and in addition satisfies

$$
\limsup _{|x| \rightarrow+\infty} \frac{\sup _{[0, T]} u(t, x)}{\Phi(x)} \leq 0
$$

hence, if $x \in \mathbb{R}^{N}$ is fixed, $\chi$ achieves its maximum at some $(\bar{t}, \bar{y})$. We claim that $(\bar{t}, \bar{y}) \rightarrow(0, x)$ as $\varepsilon \rightarrow 0$ and $C \rightarrow+\infty$. Let us fix $\varepsilon$. On denoting

$$
p_{C}:=\frac{(\bar{y}-x) \nabla \phi_{\varepsilon}(\bar{y}-x)}{\varepsilon}+\nabla \Phi(\bar{y}),
$$

the optimality conditions satisfied by the global maximum $(\bar{t}, \bar{y})$ imply that $\left(C, p_{C}\right)$ $\in \partial^{P} u(\bar{t}, \bar{y})$ and

$$
g[u]\left(\bar{t}, \bar{y}, p_{C}\right) \geq g\left[\frac{\phi_{\varepsilon}(\cdot-x)}{\varepsilon}+\Phi\right](\bar{y})=: I_{C} .
$$


By the smoothness of $\phi_{\varepsilon}$ and $\Phi$, we can see that $\lim \sup _{C \rightarrow+\infty}\left(\left|p_{C}\right|+\left|I_{C}\right|\right)<+\infty$; hence, if $C$ is sufficiently large, then

$$
C+H\left(\bar{t}, \bar{y}, u(\bar{t}, \bar{y}), p_{C}\right)+I_{C}>0 .
$$

It follows that

$$
C+H\left(\bar{t}, \bar{y}, u(\bar{t}, \bar{y}), p_{C}\right)+g[u]\left(\bar{t}, \bar{y}, p_{C}\right)>0
$$

and that necessarily $\bar{t}=0$. Moreover, since $\chi(0, \bar{y}) \geq \chi(0, x)$, we have $u(0, \bar{y}) \geq$ $u(0, x)+\Phi(\bar{y})-\Phi(x)$ and (A.3) implies that $u(0, x) \leq u_{0}(\bar{y})+\Phi(x)-\Phi(\bar{y})$. Since $u_{0}$ is usc as an upper semi-limit, we can pass to the limit as $\varepsilon \rightarrow 0$ and $C \rightarrow+\infty$ to deduce that $u(0, x) \leq u_{0}(x)$. The proof of Proposition 2.1 is now complete.

\section{A.3. Proof of the comparison principle in the sublinear case.}

Proof of Theorem 3.1. The proof proceeds in two steps. We first prove that there exists a constant $K>0$ such that

$$
\sup _{[0, T] \times \mathbb{R}^{N} \times \mathbb{R}^{N}}(u(t, x)-v(t, y)-K|x-y|)<+\infty
$$

and then prove that $u \leq v$.

Hence, let us prove (A.4). First we note that the uniform continuity of $u_{0}$ and (A2) imply that we can choose $K>0$ such that

$$
\begin{gathered}
\sup _{\mathbb{R}^{N} \times \mathbb{R}^{N}}\left(u_{0}(x)-u_{0}(y)-K|x-y|\right)<+\infty, \\
\sup _{[0, T] \times \mathbb{R}^{N} \times \mathbb{R}^{N}}(H(t, y, u(t, x), 0)-H(t, x, u(t, x), 0)-K|x-y|)<+\infty .
\end{gathered}
$$

Consider $\left(\beta_{R}\right)_{R \geq 1}$ a family of $C^{2}$ functions on $\mathbb{R}^{N}$ such that

$$
\left\{\begin{array}{l}
\text { (i) } \beta_{R} \geq 0, \\
\text { (ii) } \liminf _{|x| \rightarrow+\infty} \frac{\beta_{R}(x)}{|x|} \geq 2 R_{0}, \\
\text { (iii) }\left\|\beta_{R}\right\|_{0, \lambda^{\prime}}+\left\|\nabla \beta_{R}\right\|_{\infty}+\left\|D^{2} \beta_{R}\right\|_{\infty} \leq C \text { for } R \geq 1, \\
\text { (iv) } \lim _{R \rightarrow+\infty} \beta_{R}(x)=0 \text { for } x \in \mathbb{R}^{N},
\end{array}\right.
$$

where $R_{0}=\max \left\{\sup _{[0, T] \times \mathbb{R}^{N}} \frac{u(t, x)}{1+|x|}, \inf _{[0, T] \times \mathbb{R}^{N}} \frac{v(t, x)}{1+|x|}, 0\right\}$ and $C$ is some positive constant which does not depend on $R$. In view of the assumption (A3), the estimates (A.6), (A.7)-(iii) and Lemma B.0.1, there exists a constant $\sigma$ such that for all $R \geq 1$, $x, y \in \mathbb{R}^{N}, p \in B_{K e^{T}}$, and $t \in[0, T]$,

$$
\begin{aligned}
H\left(t, y, u(t, x), p+\nabla \beta_{R}(y)\right)-H\left(t, x, u(t, x), p-\nabla \beta_{R}(x)\right) \\
-K|x-y|-g\left[\beta_{R}\right](x)-g\left[\beta_{R}\right](y) \leq \sigma .
\end{aligned}
$$

Define

$$
M_{R}:=\sup _{[0, T] \times \mathbb{R}^{N} \times \mathbb{R}^{N}}\left(u(t, x)-v(t, y)-K e^{t}\left(1+|x-y|^{2}\right)^{1 / 2}-\beta_{R}(x)-\beta_{R}(y)-\sigma t\right) .
$$

There exists $(\bar{t}, \bar{x}, \bar{y}) \in[0, T] \times \mathbb{R}^{N} \times \mathbb{R}^{N}$ where the supremum is attained. Now either (A.4) holds true or $\lim _{R \rightarrow+\infty} M_{R}=+\infty$. In the latter case, $M_{R}$ is in particular greater than 0 and greater than the supremum in (A.5) for $R$ large enough. It follows that $u(\bar{t}, \bar{x})>v(\bar{t}, \bar{y})$ and $\bar{t} \neq 0$. By Lemma 2.3.2, there exist $a, b \in \mathbb{R}$ such that

$$
\begin{array}{r}
\left(a, p+\nabla \beta_{R}(\bar{x})\right) \in \bar{\partial}^{P} u(\bar{t}, \bar{x}), \\
\left(b, p-\nabla \beta_{R}(\bar{y})\right) \in \bar{\partial}_{P} v(\bar{t}, \bar{y}),
\end{array}
$$


with $a-b=K e^{\bar{t}}\left(1+|\bar{x}-\bar{y}|^{2}\right)^{1 / 2}+\sigma, p=K e^{\bar{t}} \frac{(\bar{x}-\bar{y})}{\left(1+|\bar{x}-\bar{y}|^{2}\right)^{1 / 2}}$ and

$$
g[v]\left(\bar{t}, \bar{y}, p-\nabla \beta_{R}(\bar{y})\right)-g[u]\left(\bar{t}, \bar{x}, p+\nabla \beta_{R}(\bar{x})\right) \leq-g\left[\beta_{R}\right](\bar{x})-g\left[\beta_{R}\right](\bar{y}) .
$$

Since $u$ is a subsolution and $v$ is a supersolution of (1.1), Lemma 2.3.1) gives

$$
\begin{aligned}
a+H\left(\bar{t}, \bar{x}, u(\bar{t}, \bar{x}), p+\nabla \beta_{R}(\bar{x})\right)+g[u]\left(\bar{t}, \bar{x}, p+\nabla \beta_{R}(\bar{x})\right) & \leq 0 \\
b+H\left(\bar{t}, \bar{y}, v(\bar{t}, \bar{y}), p-\nabla \beta_{R}(\bar{y})\right)+g[v]\left(\bar{t}, \bar{x}, p-\nabla \beta_{R}(\bar{y})\right) & \geq 0 .
\end{aligned}
$$

Subtracting the two inequalities, it becomes

$$
\begin{aligned}
\sigma \leq H\left(\bar{t}, \bar{y}, v(\bar{t}, \bar{y}), p-\nabla \beta_{R}(\bar{y})\right)-H\left(\bar{t}, \bar{x}, u(\bar{t}, \bar{x}), p+\nabla \beta_{R}(\bar{x})\right) \\
-K|\bar{x}-\bar{y}|-g\left[\beta_{R}\right](\bar{x})-g\left[\beta_{R}\right](\bar{y}) .
\end{aligned}
$$

Since $u(\bar{t}, \bar{x})>v(\bar{t}, \bar{y})$ and $H$ is strictly non-decreasing wrt $u$ (see $\left(\mathrm{A}^{\prime}\right)$ ), we find that

$$
\begin{array}{r}
\sigma<H\left(\bar{t}, \bar{y}, u(\bar{t}, \bar{x}), p-\nabla \beta_{R}(\bar{y})\right)-H\left(\bar{t}, \bar{x}, u(\bar{t}, \bar{x}), p+\nabla \beta_{R}(\bar{x})\right) \\
-K|\bar{x}-\bar{y}|-g\left[\beta_{R}\right](\bar{x})-g\left[\beta_{R}\right](\bar{y}) .
\end{array}
$$

By (A.8), we get a contradiction, and this implies that (A.4) holds true.

With this information in hand we repeat the above line of arguments to show that

$$
M:=\sup _{[0, T] \times \mathbb{R}^{N} \times \mathbb{R}^{N}}(u-v) \leq 0 .
$$

Let $\lambda^{\prime \prime} \in\left(\lambda^{\prime}, \lambda\right)$ be fixed. Consider two parameters $\varepsilon, \eta>0$ and define

$$
M_{\varepsilon, \eta}:=\sup _{[0, T] \times \mathbb{R}^{N} \times \mathbb{R}^{N}}\left(u(t, x)-v(t, y)-\frac{|x-y|^{2}}{2 \varepsilon}-\eta \frac{\left(1+|x|^{2}\right)^{\frac{\lambda^{\prime \prime}}{2}}}{\lambda^{\prime \prime}}\right) .
$$

There exists $(\bar{t}, \bar{x}, \bar{y}) \in[0, T] \times \mathbb{R}^{N} \times \mathbb{R}^{N}$, where the supremum is attained. If $M>0$, then for $\varepsilon$ and $\eta$ small enough, $M_{\varepsilon, \eta} \geq M / 2>0$ and $\bar{t} \neq 0$; indeed,

$$
M_{\varepsilon, \eta} \geq \sup _{[0, T] \times \mathbb{R}^{N} \times \mathbb{R}^{N}}\left(u(t, x)-v(t, x)-\eta \frac{\left(1+|x|^{2}\right)^{\frac{\lambda^{\prime \prime}}{2}}}{\lambda^{\prime \prime}}\right) \rightarrow M
$$

as $\eta \rightarrow 0$ and

$$
\begin{aligned}
\sup _{\mathbb{R}^{N} \times \mathbb{R}^{N}}\left(u(0, x)-v(0, y)-\frac{|x-y|^{2}}{2 \varepsilon}\right. & \left.-\eta \frac{\left(1+|x|^{2}\right)^{\frac{\lambda^{\prime \prime}}{2}}}{\lambda^{\prime \prime}}\right) \\
& \leq \sup _{\mathbb{R}^{N} \times \mathbb{R}^{N}}\left(u_{0}(x)-u_{0}(y)-\frac{|x-y|^{2}}{2 \varepsilon}\right) \rightarrow 0
\end{aligned}
$$

as $\varepsilon \rightarrow 0$, thanks to the uniform continuity of $u_{0}$. It follows that $u(\bar{t}, \bar{x})-v(\bar{t}, \bar{y}) \geq$ $M / 2$ and by (A.4):

$$
\begin{gathered}
\lim _{\varepsilon \rightarrow 0} \limsup _{\eta \rightarrow 0} \frac{|\bar{x}-\bar{y}|^{2}}{\varepsilon}=0 \\
\frac{|\bar{x}-\bar{y}|^{2}}{4 \varepsilon}+\eta \frac{\left(1+|\bar{x}|^{2}\right)^{\frac{\lambda^{\prime \prime}}{2}}}{\lambda^{\prime \prime}} \leq u(\bar{t}, \bar{x})-v(\bar{t}, \bar{y})-\frac{|\bar{x}-\bar{y}|^{2}}{4 \varepsilon} \leq C(1+\varepsilon)
\end{gathered}
$$


for some positive constant $C$. By Lemma 2.3.2, there exist

$$
\begin{array}{r}
\left(a, p+\eta\left(1+|\bar{x}|^{2}\right)^{\frac{\lambda^{\prime \prime}-2}{2}} \bar{x}\right) \in \bar{\partial}^{P} u(\bar{t}, \bar{x}), \\
(a, p) \in \bar{\partial}_{P} v(\bar{t}, \bar{y})
\end{array}
$$

with $p=\frac{\bar{x}-\bar{y}}{\varepsilon}$ and

$$
g[v](\bar{t}, \bar{y}, p)-g[u]\left(\bar{t}, \bar{x}, p+\eta\left(1+|\bar{x}|^{2}\right)^{\frac{\lambda^{\prime \prime}-2}{2}} \bar{x}\right) \leq \frac{\eta}{\lambda^{\prime \prime}} g\left[\left(1+|\cdot|^{2}\right)^{\frac{\lambda^{\prime \prime}}{2}}\right](\bar{x}) .
$$

Substracting the corresponding viscosity inequalities and using assumption (A1') (see Remark 3.1), we get

$$
\begin{gathered}
M / 2 \leq H(\bar{t}, \bar{y}, u(\bar{t}, \bar{x}), p)-H\left(\bar{t}, \bar{x}, u(\bar{t}, \bar{x}), p+\eta\left(1+|\bar{x}|^{2}\right)^{\frac{\lambda^{\prime \prime}-2}{2}} \bar{x}\right) \\
-\frac{\eta}{\lambda^{\prime \prime}} g\left[\left(1+|\cdot|^{2}\right)^{\frac{\lambda^{\prime \prime}}{2}}\right](\bar{x}) .
\end{gathered}
$$

If $\eta \leq 1$, then (A.10) implies that $p+\eta\left(1+|\bar{x}|^{2}\right)^{\frac{\lambda^{\prime \prime}-2}{2}} \bar{x} \in B_{R_{\varepsilon}}$ with

$$
R_{\varepsilon}=\sqrt{\frac{C(1+\varepsilon)}{4 \varepsilon}}+\left(\lambda^{\prime \prime} C(1+\varepsilon)\right)^{\frac{\lambda^{\prime \prime}-1}{\lambda^{\prime \prime}}} .
$$

By (A3),

$$
\begin{aligned}
M / 2 \leq & H(\bar{t}, \bar{y}, u(\bar{t}, \bar{x}), p)-H(\bar{t}, \bar{x}, u(\bar{t}, \bar{x}), p)+m_{R_{\varepsilon}}\left(\eta^{\frac{1}{\lambda^{\prime \prime}}}\left(\lambda^{\prime \prime} C(1+\varepsilon)\right)^{\frac{\lambda^{\prime \prime}-1}{\lambda^{\prime \prime}}}\right) \\
& -\frac{\eta}{\lambda^{\prime \prime}} g\left[\left(1+|\cdot|^{2}\right)^{\frac{\lambda^{\prime \prime}}{2}}\right](\bar{x}) .
\end{aligned}
$$

Assumption (A2) now gives

$$
\begin{aligned}
M / 2 \leq & m\left(|\bar{x}-\bar{y}|+\frac{|\bar{x}-\bar{y}|^{2}}{\varepsilon}\right)+m_{R_{\varepsilon}}\left(\eta^{\frac{1}{\lambda^{\prime \prime}}}\left(\lambda^{\prime \prime} C(1+\varepsilon)\right)^{\frac{\lambda^{\prime \prime}-1}{\lambda^{\prime \prime}}}\right) \\
& -\frac{\eta}{\lambda^{\prime \prime}} g\left[\left(1+|\cdot|^{2}\right)^{\frac{\lambda^{\prime \prime}}{2}}\right](\bar{x}) .
\end{aligned}
$$

By Lemma B.0.1 and A.9 , we see that the right-hand side of the inequality above tends to 0 as $\eta \rightarrow 0$ and $\varepsilon \rightarrow 0$ successively. This gives us the desired contradiction $0<M / 2 \leq 0$ and this completes the proof of Theorem 3.1.

\section{Appendix B. A teChNicAl RESUlt}

Define for $\beta \in(1,2)$ :

$$
C_{\beta}^{2}\left(\mathbb{R}^{N}\right):=\left\{f \in C_{\beta}^{0,1}\left(\mathbb{R}^{N}\right) \cap C_{b}^{2}\left(\mathbb{R}^{N}\right): D^{2} f(x)=\mathcal{O}\left(|x|^{\beta-2}\right) \text { as }|x| \rightarrow+\infty\right\} .
$$

Lemma B.0.1. Let $\lambda \in(0,2)$ and $\lambda^{\prime \prime} \in(0, \lambda)$. Then:

- If $\lambda^{\prime \prime}>1$, then $g: C_{\lambda^{\prime \prime}}^{2}\left(\mathbb{R}^{N}\right) \rightarrow C_{b}\left(\mathbb{R}^{N}\right)$.

- If $\lambda^{\prime \prime} \leq 1$, then $g: C_{b}^{2}\left(\mathbb{R}^{N}\right) \rightarrow C_{b}\left(\mathbb{R}^{N}\right)$.

Proof. Let $f \in C_{b}^{2}\left(\mathbb{R}^{N}\right)$ with $\lambda^{\prime \prime} \in(0,1]$. By a second order expansion, we get

$$
\begin{aligned}
& |g[f]|(x) \leq \sup _{B_{r}(x)}\left|D^{2} f\right| \int_{B_{r}}|z|^{2} d \mu(z) d \tau+\sup _{B_{r}(x)^{c}}|\nabla f| \int_{B_{r}^{c}} \frac{|z|}{1+|z|^{2}} d \mu(z) \\
& \quad+\sup _{B_{r}(x)^{c}}|f| \int_{B_{r}^{c}} d \mu(z)
\end{aligned}
$$

for all $r \geq 0$ and all $x \in \mathbb{R}^{N}$. The proof is complete in this case. 
If now $\lambda^{\prime \prime} \in(1,2)$, write

$$
g[f](x)=-\int_{B_{r}}\left(f(x+z)-f(x)-\nabla f(x) \cdot \frac{z}{1+|z|^{2}}\right) d \mu(z)-\int_{\mathbb{R}^{N} \backslash B_{r}}(\ldots) d \mu(z)
$$

for $x \in \mathbb{R}^{N} \backslash B_{1}$ and choose $r=|x| / 2$. Observe that

$$
\sup _{z^{\prime} \in B_{|x| / 2}}\left(1+\left|x+z^{\prime}\right|\right)^{\lambda^{\prime \prime}-2} \leq(1+3|x| / 2)^{\lambda^{\prime \prime}-2}
$$

so that

$$
\begin{aligned}
|g[f](x)| \leq & C(1+3|x| / 2)^{\lambda^{\prime \prime}-2} \int_{B_{|x| / 2}}|z|^{2} d \mu(z)+C(1+|x|)^{\lambda^{\prime \prime}-1} \int_{\mathbb{R}^{N} \backslash B_{|x| / 2}} \frac{|z|}{1+|z|^{2}} d \mu(z) \\
& +C \int_{\mathbb{R}^{N} \backslash B_{|x| / 2}}(1+|z|)^{\lambda^{\prime \prime}-1} \frac{|z|}{1+|z|^{2}} d \mu(z)+C \int_{\mathbb{R}^{N} \backslash B_{|x| / 2}}|z|^{\lambda^{\prime \prime}} d \mu(z) \\
\leq & C(1+|x|)^{\lambda^{\prime \prime}-2} J_{2}+C(1+|x|)^{\lambda^{\prime \prime}-1} I_{-1}+C I_{\lambda^{\prime \prime}-2}+C I_{\lambda^{\prime \prime}},
\end{aligned}
$$

where

$$
J_{2}=\int_{|z| \leq|x| / 2}|z|^{2} \mu(d z)=C \int_{0}^{|x| / 2} r^{1-\lambda} d r \leq C\left(1+|x|^{2-\lambda}\right),
$$

and for $\alpha=-1, \lambda^{\prime \prime}-2, \lambda^{\prime \prime}$ (hence $\alpha<\lambda$ )

$$
\begin{aligned}
I_{\alpha} & =\int_{\mathbb{R}^{N} \backslash B_{|x| / 2}}|z|^{\alpha} d \mu(z)=C \int_{|x| / 2}^{+\infty} r^{\alpha} r^{N-1} r^{-N-\lambda} d r \\
& =C \int_{|x| / 2}^{+\infty} r^{\alpha-1-\lambda} d r=C|x|^{\alpha-\lambda} .
\end{aligned}
$$

Hence, we obtain for $x \in \mathbb{R}^{N} \backslash B_{1}$

$$
|g[f](x)| \leq C+C|x|^{\lambda^{\prime \prime}-\lambda}+C|x|^{\lambda^{\prime \prime}-2-\lambda} \leq C .
$$

This completes the proof of the lemma.

\section{Appendix C. Different sets of Assumptions}

$H$ continuous, $\lambda \in(0,2)$ and $\lambda^{\prime} \in(0, \lambda)$.

- The sublinear Case

(A1) There exists $\nu \in \mathbb{R}$ such that for all $x \in \mathbb{R}^{N}, u, v \in \mathbb{R}, u \leq v, p \in \mathbb{R}^{N}$, and $t \in[0, T]$,

$$
H(t, x, v, p)-H(t, x, u, p) \geq \nu(v-u) .
$$

$\left(\mathrm{A}^{\prime}\right)$ For all $x \in \mathbb{R}^{N}, u, v \in \mathbb{R}, u \leq v, p \in \mathbb{R}^{N}$, and $t \in[0, T]$,

$$
H(t, x, v, p)-H(t, x, u, p) \geq v-u .
$$

(A2) There exists a modulus of continuity $m$ such that for all $x, y \in \mathbb{R}^{N}$, $u \in \mathbb{R}, p \in \mathbb{R}^{N}$, and $t \in[0, T]$,

$$
|H(t, x, u, p)-H(t, y, u, p)| \leq m((1+|p|)|x-y|) .
$$

$\left(\mathrm{A} 2^{\prime}\right)$ There exists a constant $C_{1}$ such that for all $x, y \in \mathbb{R}^{N}, u \in \mathbb{R}, p \in \mathbb{R}^{N}$, and $t \in[0, T]$,

$$
|H(t, x, u, p)-H(t, y, u, p)| \leq C_{1}(1+|p|)|x-y| .
$$


(A3) For all $R>0$, there exists a modulus of continuity $m_{R}$ such that for all $x \in \mathbb{R}^{N}, u \in \mathbb{R}, p, q \in B_{R}$, and $t \in[0, T]$,

$$
|H(t, x, u, p)-H(t, x, u, q)| \leq m_{R}(|p-q|) .
$$

$\left(\mathrm{A} 3^{\prime}\right)$ For all $R>0$, there exists a constant $C_{R}$ such that for all $x \in \mathbb{R}^{N}$, $u, v \in \mathbb{R}, p, q \in B_{R}$, and $t \in[0, T]$,

$$
|H(t, x, u, p)-H(t, x, v, q)| \leq C_{R}(|u-v|+|p-q|) .
$$

(A4) There exists a constant $C_{0}$ such that for all $x \in \mathbb{R}^{N}$,

$$
\sup _{[0, T]}|H(t, x, 0,0)| \leq C_{0}\left(1+|x|^{\lambda^{\prime}}\right) .
$$

- The Superlinear CASE

(B1) There exists a constant $C_{3}$ such that for all $x \in \mathbb{R}^{N}, u \in \mathbb{R}, p \in \mathbb{R}^{N}$, and $t \in[0, T]$,

$$
|H(t, x, u, p)-H(t, x, v, p)| \leq C_{3}|u-v| .
$$

(B2) For all $R>0$, there exists a modulus of continuity $m_{R}$ such that for all $x, y \in B_{R}, u \in[-R, R], p \in \mathbb{R}^{N}$, and $t \in[0, T]$,

$$
|H(t, x, u, p)-H(t, y, u, p)| \leq m_{R}((1+|p|)|x-y|) .
$$

(B2') There exists a constant $C_{1}$ such that for all $x, y \in \mathbb{R}^{N}, u \in \mathbb{R}, p \in \mathbb{R}^{N}$, and $t \in[0, T]$,

$|H(t, x, u, p)-H(t, y, u, p)| \leq C_{1}\left(1+|x|^{\lambda^{\prime}-1}+|y|^{\lambda^{\prime}-1}+|p|\right)|x-y|$.

(B3) There exists a modulus of continuity $m$ such that for all $x \in \mathbb{R}^{N}$, $u \in \mathbb{R}, p, q \in \mathbb{R}^{N}$, and $t \in[0, T]$,

$$
|H(t, x, u, p)-H(t, x, u, q)| \leq m((1+|x|)|p-q|) .
$$

(B3') There exists a constant $C_{2}$ such that for all $x, y \in \mathbb{R}^{N}, u \in \mathbb{R}, p, q \in$ $\mathbb{R}^{N}$, and $t \in[0, T]$,

$$
|H(t, x, u, p)-H(t, x, u, q)| \leq C_{2}(1+|x|)|p-q| .
$$

- The uniformly integrable CaSe

(C2) The Hamiltonian $H$ is locally Lipschitz continuous wrt $x$, and there are a constant $C_{1}$ and a locally bounded function $\Theta: \mathbb{R}^{N} \rightarrow \mathbb{R}^{+}$such that $\int_{\mathbb{R}^{N}} \sup _{B_{R}(z)} \Theta^{2} d \mu_{b}(z)<+\infty$, for some $R>0$, and for a.e. $x \in \mathbb{R}^{N}$, $u \in \mathbb{R}, p \in \mathbb{R}^{N}$, and $t \in[0, T]$,

$$
\left|\nabla_{x} H(t, x, u, p)\right| \leq \Theta(x)+C_{1}|p| .
$$

(C3) There exists a modulus of continuity $m$ such that for all $x, y \in \mathbb{R}^{N}$, $p, q \in \mathbb{R}^{N}, u \in \mathbb{R}$, and $t \in[0, T]$,

$$
|H(t, x, u, p)-H(t, x, u, q)| \leq m(|p-q|) .
$$

(C3') There exists a constant $C_{2}$ such that for all $x, y \in \mathbb{R}^{N}, u \in \mathbb{R}, p, q \in$ $\mathbb{R}^{N}$, and $t \in[0, T]$,

$$
|H(t, x, u, p)-H(t, x, u, q)| \leq C_{2}|p-q| .
$$

(C4) There exists $R>0$ such that

$$
\int_{\mathbb{R}^{N}[0, T] \times B_{R}(z)}|H(\cdot, \cdot, 0,0)| d \mu_{b}(z)<+\infty .
$$




\section{- The Assumption For the STRONG CONVERGENCE OF GRADIEnts}

(D) The non-linearity $H$ is strictly convex wrt $p$.

\section{ACKNOWLEDGEMENT}

The authors thank the referee for a careful reading and meaningful remarks that yielded a substantially improved version of this paper.

\section{REFERENCES}

1. Nathaël Alibaud, Entropy formulation for fractal conservation laws, J. Evol. Equ. 7 (2006), no. 1, 145-175. MR2305729

2. Nathaël Alibaud, Jérôme Droniou, and Julien Vovelle, Occurrence and non-appearance of shocks in fractal Burgers equations, J. Hyperbolic Differ. Equ. 4 (2007), no. 3, 479-499. MR 2339805

3. O. Alvarez and A. Tourin, Viscosity solutions of nonlinear integro-differential equations, Ann. Inst. H. Poincaré Anal. Non Linéaire 13 (1996), no. 3, 293-317. MR1395674 (97d:35023)

4. O. Alvarez, P. Hoch, Y. Le Bouar, and R. Monneau, Résolution en temps court d'une équation de Hamilton-Jacobi non locale décrivant la dynamique d'une dislocation, C. R. Math. Acad. Sci. Paris 338 (2004), no. 9, 679-684. MR2065373 (2005b:35021)

5. G. Barles and C. Imbert, Second-order elliptic integro-differential equations: Viscosity solutions' theory revisited, Annales de l'IHP 25 (2008), no. 3, 567-585. MR2422079

6. G. Barles and B. Perthame, Exit time problems in optimal control and vanishing viscosity method, SIAM J. Control Optim. 26 (1988), no. 5, 1133-1148. MR957658 (89i:49021)

7. Guy Barles, Solutions de viscosité des équations de Hamilton-Jacobi, Mathématiques \& Applications (Berlin) [Mathematics \& Applications], vol. 17, Springer-Verlag, Paris, 1994. MR 1613876 (2000b:49054)

8. Guy Barles, Rainer Buckdahn, and Etienne Pardoux, Backward stochastic differential equations and integral-partial differential equations, Stochastics Stochastics Rep. 60 (1997), no. 12, 57-83. MR98d:60110

9. A. Bensoussan and J.-L. Lions, Contrôle impulsionnel et inéquations quasi variationnelles, Méthodes Mathématiques de l'Informatique [Mathematical Methods of Information Science], vol. 11, Gauthier-Villars, Paris, 1982. MR673169 (84g:49012)

10. Piotr Biler, Tadahisa Funaki, and Wojbor A. Woyczynski, Fractal Burgers equations, J. Differential Equations 148 (1998), no. 1, 9-46. MR1637513 (99g:35111)

11. Piotr Biler, Grzegorz Karch, and Wojbor A. Woyczynski, Asymptotics for multifractal conservation laws, Studia Math. 135 (1999), no. 3, 231-252. MR.1708995 (2000h:35098)

12. Krzysztof Bogdan and Tomasz Jakubowski, Estimates of heat kernel of fractional Laplacian perturbed by gradient operators, Comm. Math. Phys. 271 (2007), no. 1, 179-198. MR2283957 (2007k:47076)

13. P. Clavin, Diamond patterns in the cellular front of an overdriven detonation, Phys. Rev. Letters 88 (2002).

14. Peter Constantin, Andrew J. Majda, and Esteban Tabak, Formation of strong fronts in the 2-D quasigeostrophic thermal active scalar, Nonlinearity $\mathbf{7}$ (1994), no. 6, 1495-1533. MR 1304437 (95i:76107)

15. R. Cont and P. Tankov, Financial modelling with jump processes, Chapman \& Hall/CRC Financial Mathematics Series, Chapman \& Hall/CRC, Boca Raton, FL, 2004. MR2042661 (2004m:91004)

16. Philippe Courrège, Sur la forme intégro-différentielle des opérateurs de $c_{k}^{\infty}$ dans c satisfaisant au principe du maximum, Séminaire BRELOT-CHOQUET-DENY, théorie du potentiel, vol. 10, 1965/1966, 10ème année, pp. 1-28.

17. Michael G. Crandall, Hitoshi Ishii, and Pierre-Louis Lions, User's guide to viscosity solutions of second order partial differential equations, Bull. Amer. Math. Soc. (N.S.) 27 (1992), no. 1, 1-67. MR 92j:35050

18. Gianni Dal Maso and François Murat, Almost everywhere convergence of gradients of solutions to nonlinear elliptic systems, Nonlinear Anal. 31 (1998), no. 3-4, 405-412. MR1487552 (99d:35046) 
19. J. Droniou, T. Gallouet, and J. Vovelle, Global solution and smoothing effect for a non-local regularization of a hyperbolic equation, J. Evol. Equ. 3 (2003), no. 3, 499-521. MR2019032

20. N. Forcadel, C. Imbert, and R. Monneau, Homogenization of some systems of particles with two-body interactions and of the dislocation dynamics, Discrete and Continuous Dynamical Systems (to appear).

21. M. G. Garroni and J.-L. Menaldi, Green functions for second order parabolic integrodifferential problems, Pitman Research Notes in Mathematics Series, vol. 275, Longman Scientific \& Technical, Harlow, 1992. MR1202037 (94g:35110)

22. Maria Giovanna Garroni and Jose Luis Menaldi, Second order elliptic integro-differential problems, Chapman \& Hall/CRC Research Notes in Mathematics, vol. 430, Chapman \& Hall/CRC, Boca Raton, FL, 2002. MR1911531 (2003i:35002)

23. F. Gimbert and P.-L. Lions, Existence and regularity results for solutions of second-order, elliptic integro-differential operators, Ricerche Mat. 33 (1984), no. 2, 315-358. MR810193 (87d:45022)

24. I. Held, R. Pierrehumbert, S Garner, and K. Swanson, Surface quasi-geostrophic dynamics, J. of Fluid Mech. 282 (1995), 1-20. MR.1312238 (95i:76017)

25. C. Imbert, A non-local regularization of first order Hamilton-Jacobi equations, J. Differential Equations 211 (2005), no. 1, 218-246. MR2121115 (2006e:35038)

26. Hitoshi Ishii, Uniqueness of unbounded viscosity solution of Hamilton-Jacobi equations, Indiana Univ. Math. J. 33 (1984), no. 5, 721-748. MR85h:35057

27. , Perron's method for Hamilton-Jacobi equations, Duke Math. J. 55 (1987), no. 2, 369-384. MR894587 (89a:35053)

28. E. R. Jakobsen and K. H. Karlsen, Continuous dependence estimates for viscosity solutions of integro-PDEs, J. Differential Equations 212 (2005), no. 2, 278-318. MR2129093 $(2005 \mathrm{k}: 35198)$

29. A. N. Kolmogorov, I. G. Petrovsky, and Piskunov N. S., Etude de l'équation de la diffusion avec croissance de la quantité de matière et son application à un problème biologique, Bulletin de l'Université d'Etat de Moscou (1937), pp. 1-26, English translation: Study of the diffusion Equation with growth of the quantity of matter and its application to a biological problem.

30. Pierre-Louis Lions, Generalized solutions of Hamilton-Jacobi equations, Research Notes in Mathematics, vol. 69, Pitman (Advanced Publishing Program), Boston, Mass., 1982. MR.84a:49038

31. J. D. Murray, Mathematical biology, second ed., Biomathematics, vol. 19, Springer-Verlag, Berlin, 1993. MR:1239892 (94j:92002)

32. J. Pedlovsky, Geophysical Fluid Dynamics, Springer-Verlag, 1987.

33. A. Sayah, Équations d'Hamilton-Jacobi du premier ordre avec termes intégro-différentiels. I. Unicité des solutions de viscosité. II. Existence de solutions de viscosité., Comm. Partial Differential Equations 16 (1991), no. 6-7, 1057-1093. MR1116853(93b:35027a)

34. H. M. Soner, Optimal control of jump-Markov processes and viscosity solutions, Stochastic differential systems, stochastic control theory and applications (Minneapolis, Minn., 1986), IMA Vol. Math. Appl., vol. 10, Springer, New York, 1988, pp. 501-511. MR934740|(89h:93055)

35. Wojbor A. Woyczyński, Lévy processes in the physical sciences, Lévy processes, Birkhäuser Boston, Boston, MA, 2001, pp. 241-266. MR2002d:82029

UFR Sciences et techniques, Université de Franche-Comté, 16 route de Gray, 25030 BESANCON CEDEX, France E-mail address: Nathael.Alibaud@ens2m.fr

Centre De Recherche en Mathématiques de la Décision, Université Paris-Dauphine, Pl. De Lattre de Tassigny, 75775 Paris Cedex 16, France

E-mail address: imbert@ceremade.dauphine.fr 